\title{
Geometric Control of Universal Hydrodynamic Flow in a Two-Dimensional Electron Fluid
}

\author{
Aydın Cem Keser, ${ }^{1,2, *}$ Daisy Q. Wang $\odot,{ }^{1,2, *}$ Oleh Klochan, ${ }^{3,2}$ Derek Y. H. Ho, ${ }^{4}$ Olga A. Tkachenko, ${ }^{5}$ \\ Vitaly A. Tkachenko, ${ }^{5,6}$ Dimitrie Culcer, ${ }^{1,2}$ Shaffique Adam $\odot,{ }^{4,7,8}$ Ian Farrer®, ${ }^{9, \dagger}$ David A. Ritchie, ${ }^{9}$ \\ Oleg P. Sushkov, ${ }^{1,2}$ and Alexander R. Hamilton ${ }^{1,2}$ \\ ${ }^{1}$ School of Physics, University of New South Wales, Sydney, New South Wales 2052, Australia \\ ${ }^{2}$ Australian Research Council Centre of Excellence in Future Low-Energy Electronics Technologies, \\ University of New South Wales, Sydney 2052, Australia \\ ${ }^{3}$ School of Science, University of New South Wales, Canberra, \\ Australian Capital Territory 2612, Australia \\ ${ }^{4}$ Yale-NUS College, 16 College Avenue West, 138614, Singapore \\ ${ }^{5}$ Rzhanov Institute of Semiconductor Physics of SB RAS, Novosibirsk, 630090, Russia \\ ${ }^{6}$ Novosibirsk University, Novosibirsk, 630090, Russia \\ ${ }^{7}$ Centre for Advanced 2D Materials and Graphene Research Centre, National University of Singapore, \\ 6 Science Drive 2, 117546, Singapore \\ ${ }^{8}$ Department of Physics, Faculty of Science National University of Singapore, \\ Science Drive 3, 117551, Singapore \\ ${ }^{9}$ Cavendish Laboratory, J. J. Thomson Avenue, Cambridge, CB3 OHE, United Kingdom
}

(Received 16 March 2021; revised 20 May 2021; accepted 22 June 2021; published 6 August 2021)

\begin{abstract}
Fluid dynamics is one of the cornerstones of modern physics and has recently found applications in the transport of electrons in solids. In most solids, electron transport is dominated by extrinsic factors, such as sample geometry and scattering from impurities. However, in the hydrodynamic regime, Coulomb interactions transform the electron motion from independent particles to the collective motion of a viscous "electron fluid." The fluid viscosity is an intrinsic property of the electron system, determined solely by the electron-electron interactions. Resolving the universal intrinsic viscosity is challenging, as it affects the resistance only through interactions with the sample boundaries, whose roughness not only is unknown but also varies from device to device. Here, we eliminate all unknown parameters by fabricating samples with smooth sidewalls to achieve the perfect slip boundary condition, which has been elusive in both molecular fluids and electronic systems. We engineer the device geometry to create viscous dissipation and reveal the true intrinsic hydrodynamic properties of a $2 \mathrm{D}$ system. We observe a clear transition from ballistic to hydrodynamic electron motion, driven by both temperature and magnetic field. We directly measure the viscosity and electron-electron scattering lifetime (the Fermi quasiparticle lifetime) over a wide temperature range without fitting parameters and show they have a strong dependence on electron density that cannot be explained by conventional theories based on the random phase approximation.
\end{abstract}

DOI: 10.1103/PhysRevX.11.031030

\section{INTRODUCTION}

Fluid dynamics is one of cornerstones of modern physics and technology, with wide-ranging applications. Although a

\footnotetext{
*These authors contributed equally.

${ }^{\dagger}$ Present address: Department of Electronic and Electrical Engineering, The University of Sheffield, Mappin Street, Sheffield, S1 3JD, United Kingdom.

Published by the American Physical Society under the terms of the Creative Commons Attribution 4.0 International license. Further distribution of this work must maintain attribution to the author(s) and the published article's title, journal citation, and DOI.
}

\author{
Subject Areas: Condensed Matter Physics, \\ Fluid Dynamics, \\ Semiconductor Physics
}

well-established subject (Bernoulli's law was formulated in 1738), it has important modern manifestations such as hydrodynamics of the quark-gluon plasma and of electrons in solids. While the dynamics of fluids are universal and depend only on the viscosity, the boundary conditions between the fluid and the containing solid play a crucial role. These boundary conditions are nonuniversal and depend on the details of the solid surface, the fluid, ambient conditions, and the structure of complex boundary layers. The precise nature of fluid boundary conditions at various interfaces is a long-standing problem of great practical importance.

Hydrodynamic flow of electrons in solids occurs when extrinsic momentum-relaxing processes, such as 
electron-phonon and electron-impurity collisions, are much slower than intrinsic electron-electron scattering processes, which conserve the fluid's momentum [1,2]. Recent studies in graphene and other clean 2D systems have demonstrated viscous electron flow through Poiseuille flow [3,4], thermal and electrical transport effects [5-12], and modifications to the Hall effect $[13,14]$. However, the boundary problem remains unresolved: Most studies are performed in systems with diffusive boundaries $[3,10,15,16]$. The boundaries introduce a system-dependent unknown parameter, the "slip length" $\beta$ [17], which can vary with experimental conditions such as temperature and magnetic field, inhibiting quantitative analysis of experimental data. A perfect slip boundary condition $(\beta \rightarrow \infty)$ would eliminate this unknown, allowing a direct measurement of the viscosity and, hence, the Fermi liquid quasiparticle lifetime (since viscosity depends only on electron-electron scattering). However, the perfect slip boundary condition has been elusive and remains a mathematical idealization in the literature.

The central idea of our work is to create devices with perfectly smooth sidewalls. This work eliminates unknown boundary effects and constitutes the first realization of universal viscous flow with the perfect slip condition. We first demonstrate perfect slip boundary conditions with no viscous dissipation in straight channels and then controllably introduce viscous dissipation by carefully engineering the device geometry. The fluid flow is now determined solely by the geometry, hence bearing the name "universal hydrodynamic flow".

Using this approach, we observe a clear transition from ballistic to hydrodynamic electron motion, driven by both temperature (which is expected) and also by magnetic field (which is not). Moreover, the absence of unknown boundary conditions allows quantitative extraction of the viscosity and, hence, Fermi liquid quasiparticle lifetime over a wide temperature range, from $T \ll E_{F}$ to $T \sim E_{F}$. The experimental data reveal an unexpected and unexplained deviation of the electron-electron scattering length from existing theoretical models.

\section{HYDRODYNAMICS IN SAMPLES WITH SMOOTH BOUNDARIES}

Describing the hydrodynamic flow of a fluid has two ingredients: (i) the dynamic Navier Stokes equation and (ii) the boundary condition at the fluid-solid interface. While the former is universal, the latter is not.

We begin by considering a straight channel with perfectly smooth boundaries [18]. No matter how strong the electronelectron interactions, there is no viscous contribution in this straight channel, since the electron flow is uniform as shown in Fig. 1(c). The resistance $R_{\text {straight }}=\rho_{\text {Drude }} L / W$ arises purely from phonons and impurity scattering. Viscous (a)

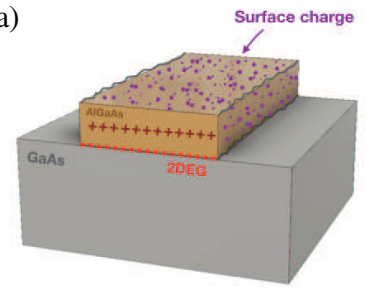

(b)

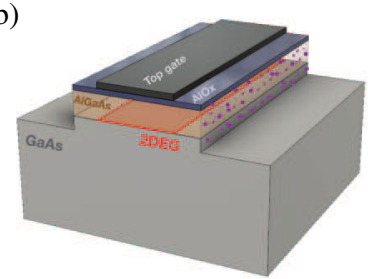

(c) Ohmic power dissipation (viscous $=0$ )



(d) Ohmic only

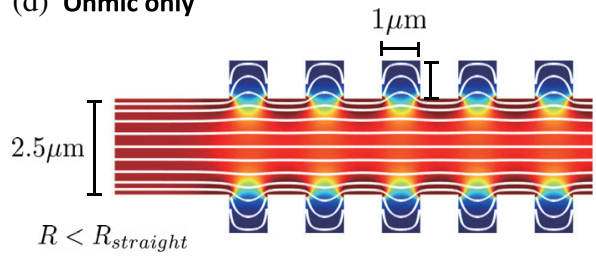

(e) Viscous only

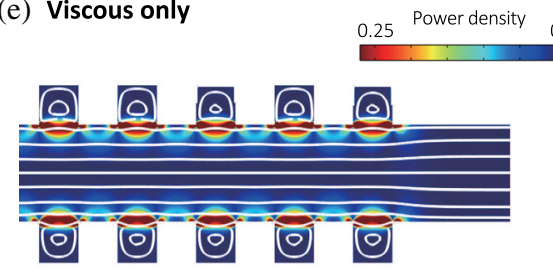

(f) Ohmic + viscous

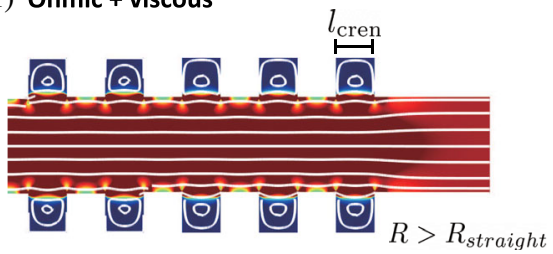

FIG. 1. (a),(b) 3D schematics showing (a) a conventional modulation-doped $\mathrm{GaAs} / \mathrm{Al}_{x} \mathrm{Ga}_{1-x}$ As heterostructure. The conduction channel is patterned using chemical etching, which causes rough sidewalls, while random surface charge on the sidewalls creates additional disorder. (b) An accumulation-mode $\mathrm{GaAs} / \mathrm{Al}_{x} \mathrm{Ga}_{1-x} \mathrm{As}$ device. The channel is defined by a metal top gate and kept away from etched sidewalls and surface charge. (c)-(f) Theoretical simulations (see the Appendix D) of the power dissipation density ( $W / \mathrm{m}^{2}$ ) under the perfect slip boundary condition (width $W=2.5 \mu \mathrm{m}$, length $L=25 \mu \mathrm{m}$ ) in the linear response regime (Reynolds number much smaller than 1). The color scale represents the magnitude of the dissipation, and white lines show the electron flow streamlines. (c) Power dissipation density of a straight channel. The dissipation is purely Ohmic, as viscous contribution vanishes with perfect slip boundaries. (d) Ohmic power dissipation density of a crenellated channel (crenellation size $l_{\text {cren }}=1 \mu \mathrm{m}$ ) with zero viscosity, i.e., nonviscous electron transport. The Ohmic resistance of the crenellated channel is smaller than the straight channel. (e) Viscous power dissipation density of the crenellated channel with a viscosity of $\nu=1.15 \times 10^{-2} \mathrm{~m}^{2} / \mathrm{s}$. The viscous power dissipation density concentrates around the regions where the streamlines deform the most to form slow whirlpools in the crenellations [19]. The viscous power dissipation vanishes near the boundaries due to the perfect slip boundary condition. (f) Total power dissipation density of the crenellated channel for a viscous electron flow summing up both Ohmic and viscous contributions. 
transport is introduced by modifying the device geometry with artificially engineered crenellations on the channel sidewalls, which causes nonuniform electron flow and, thus, viscous power dissipation as shown in Figs. 1(d)-1(f).

\section{A. Advantages of smooth boundaries}

The advantage of the smooth sidewalls with engineered structures lies in three aspects.

(i) All uncertainties associated with the slip length and boundary conditions are eliminated.

(ii) The electron transport regime can be unambiguously identified, simply by comparing the resistance of the straight channel and the crenellated channel. In transport measurements of samples with rough boundaries, it is hard to determine if the resistance $R(T)$ is due to viscous effects or scattering from extrinsic impurities and phonons. In our design, when the transport is dominated by scattering with phonons and impurities, the wider crenellated channel has a lower resistance than the straight channel $R_{\text {cren }}<R_{\text {straight. }}$. This is because the crenellated channel is on average wider for the same length. However, in the hydrodynamic regime where elec- tron-electron scattering dominates, the additional viscous contribution increases the resistance of the crenellated channel so that $R_{\text {cren }}>R_{\text {straight }}$.

(iii) Most importantly, all experimental parameters associated with phonon and impurity scattering can be quantified through a direct comparison of the resistance of the straight and crenellated channels. Even when the electron transport is deep in the hydrodynamic regime, the resistance of the straight channel does not have any viscous contribution. This allows the electron-phonon and electron-impurity scattering processes to be fully characterized, so that the electron viscosity can be directly determined from the resistance of the crenellated channel without any unknown fitting parameters.

\section{B. Experimental realization and verification of the perfect slip condition}

Experimentally, the key challenge is how to make samples with perfect slip boundaries. In most conventional devices, such as graphene or the modulation-doped GaAs/AlGaAs heterostructure shown in Fig. 1(a), chemical etching is required to pattern the channel of the $2 \mathrm{D}$ (a)

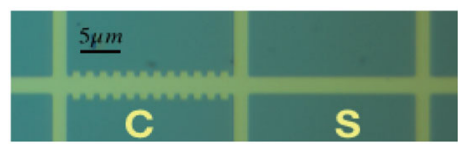

(b)

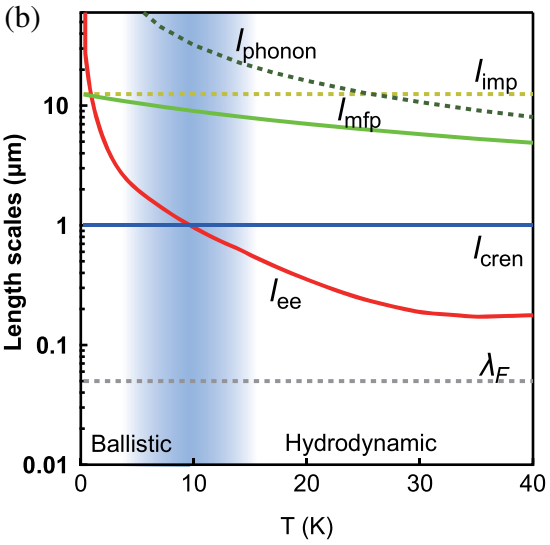

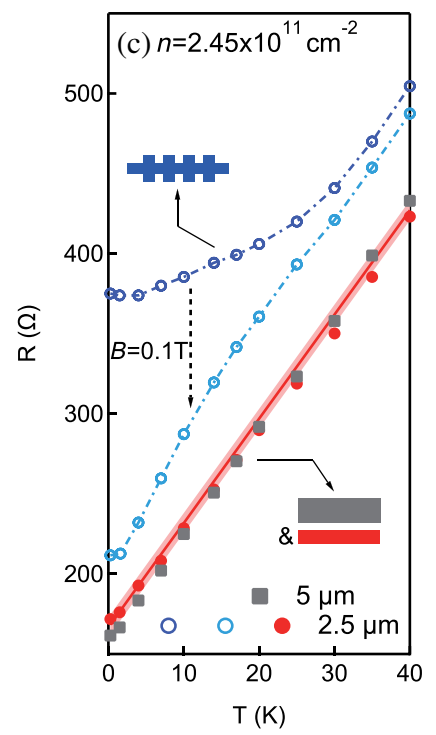
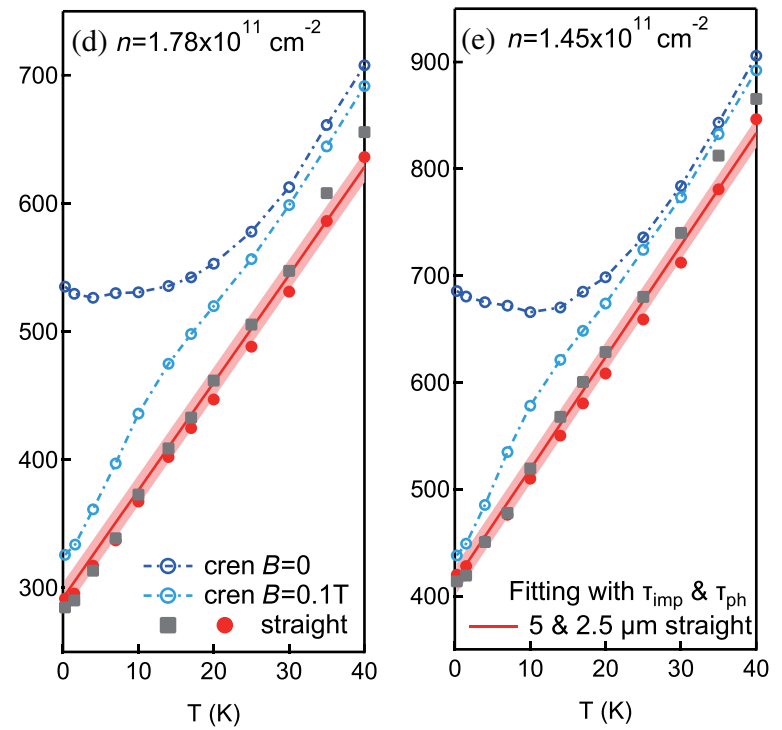

FIG. 2. (a) Image of the accumulation-mode GaAs heterostructure device containing a high-mobility 2DEG. The gate-defined channel contains two sections: a $2.5-\mu \mathrm{m}$-wide and $25-\mu \mathrm{m}$-long straight channel labeled " $S$ " and a crenellated channel " $C$ " of the same dimensions with crenellations of $l_{\text {cren }}=1 \mu \mathrm{m}$. (b) Key length scales and transport regimes in the system at $n=2.45 \times 10^{11} \mathrm{~cm}^{-2} \cdot l_{\mathrm{mfp}}$ is the momentum-relaxing mean free path due to electron-phonon $\left(l_{\text {phonon }}\right)$ and electron-impurity $\left(l_{\text {imp }}\right)$ scattering. $l_{\text {cren }}$ is the size and spacing of the square crenellations. $\lambda_{F}$ is the Fermi wavelength. $l_{e e}$ is the theoretically calculated electron-electron scattering length. The shaded blue area indicates the gradual crossover from hydrodynamic to ballistic transport regimes around $T \sim 10 \mathrm{~K}$ as the smallest length scale in the system varies between $l_{\text {cren }}$ and $l_{e e}$. (c),(d) Experimentally measured resistance of both straight and crenellated channels $R_{\text {straight }}$ and $R_{\text {cren }}$, respectively, as a function of the temperature for three electron densities $n=2.45$, 1.78, and $1.45 \times 10^{11} \mathrm{~cm}^{-2}$, respectively. The resistances of two straight channels with different widths $W=2.5$ and $5 \mu \mathrm{m}$ but the same length-to-width ratio are shown by the red solid circles and gray squares and are indistinguishable. The solid red lines are fits to $R_{\text {straight }}$ including both electron-impurity and electron-phonon scattering with the shaded area presenting the uncertainty. The resistance of the crenellated channel $C$ is shown both at $B=0$ (dark blue empty circles) and with a small out-of-plane magnetic field $B=0.1 \mathrm{~T}$ (light blue empty circles). 
system, creating microscopically rough sidewalls. Moreover, random surface charge on the sidewalls of the channel creates additional disorder at the boundary. To avoid the uncontrolled roughness and disorder that cause rough boundary conditions, we utilize accumulationmode $\mathrm{GaAs} / \mathrm{AlGaAs}$ heterostructure (W639) depicted in Fig. 1(b). There is no chemical doping in these accumulation-mode devices, and the conduction channel is induced by applying a positive bias to the metallic top gate. This technique ensures that the 2DEG is kept away from etched sidewalls and surface charge, providing a very smooth boundary.

The device used in this study is divided into multiple segments containing both straight and crenellated channels, in which the resistance of each segment can be measured independently. Figure 2(a) shows a $2.5-\mu \mathrm{m}$-wide by $25-\mu \mathrm{m}$ long straight channel, adjacent to a crenellated channel of the same length, with a minimum width varying between 2.5 and $4.5 \mu \mathrm{m}$, due to $1 \mu \mathrm{m}$ by $1 \mu \mathrm{m}$ crenellations. Figure 2(b) shows the calculated characteristic length scales for this device. For $10 \mathrm{~K} \lesssim T \lesssim 40 \mathrm{~K}$, the electron-electron scattering length $l_{e e}$ is the shortest length scale in the system, so that hydrodynamic effects are significant. At very low temperatures $T \lesssim 10 \mathrm{~K}, l_{e e}$ exceeds the characteristic length scale of the device $l_{\text {cren }} \sim 1 \mu \mathrm{m}$, and electron transport is ballistic.

We verify the smooth boundary condition in our experiment by comparing the resistance of the two straight channels with different widths: 5 and $2.5 \mu \mathrm{m}$ (both have the same length-to-width ratio of 10). The two straight channels have almost identical resistance across the whole temperature range for all three densities, shown by the red circles and gray squares in Figs. 2(c)-2(e). This result proves that the perfect slip boundary condition is satisfied in our device, since the resistance of channels with rough boundaries has a strong width dependence in both the hydrodynamic [1] and ballistic [21] regimes.

\section{DISENTANGLING DIFFUSIVE AND VISCOUS TRANSPORT}

\section{A. Straight channel}

One of the key challenges in quantitative extraction of the viscosity of the electron fluid is how to disentangle viscous and nonviscous contributions to the resistance. A unique advantage of the perfect slip boundaries is that the resistivity of the straight channels has no ballistic backscattering or viscous components and, hence, serves as an absolute reference from which all momentum-relaxing contributions can be measured: (i) From the resistance at base temperature $T=0.25 \mathrm{~K}$, we calculate the momentum relaxation length due to impurity scattering $l_{\text {imp }}=v_{F} \tau_{\text {imp }} \approx 10 \mu \mathrm{m}$, corresponding to a mobility on the order of $10^{6} \mathrm{~cm}^{2} / \mathrm{V} \mathrm{s}$ (see Appendixes $\mathrm{A}$ and B). (ii) The linear increase of $R_{\text {straight }}(T)$ with temperature is due to scattering from acoustic phonons (see Appendix C). We extract the phonon scattering time $\tau_{\mathrm{ph}}=A_{\mathrm{ph}}^{\tau} / T$ from the slope $d R_{\text {straight }}(T) / d T$. This analysis gives a phonon coupling constant of $A_{\mathrm{ph}}^{\tau}=1.5 \mathrm{~ns} \cdot \mathrm{K}$, consistent with previous studies of electrons in GaAs [16,22].

\section{B. Crenellated channel}

The resistance of the crenellated channel with a width of $2.5 \mu \mathrm{m}$ and crenellations of $l_{\text {cren }}=1 \mu \mathrm{m}$ is shown as the dark blue circles in Figs. 2(c)-2(e). The crenellated channel resistance is always higher than that of the straight channels, despite having the same length and minimum width as the 2.5- $\mu \mathrm{m}$-wide straight channel. At high temperatures, $R_{\text {cren }}(T)$ exhibits a close to linear dependence on $T$ with the same slope as $R_{\text {straight }}(T)$. As the temperature is lowered, $R_{\text {cren }}(T)$ starts to deviate from the linear dependence and rapidly increases when $T<10 \mathrm{~K}$, which is particularly visible at low electron densities. This nonmonotonic behavior of $R_{\text {cren }}(T)$ at low temperatures is caused by quasiballistic effects at low temperatures [23], which hide the viscous behavior $[3,6,7,16]$ (see Appendix $\mathrm{H}$ and Fig. 10). Disentangling the viscous, diffusive, and ballistic effects can be very complicated both theoretically and experimentally when the boundary condition is unknown $[3,6]$ but is simple for smooth boundaries. In this limit, most of the ballistic effects can be excluded by applying a small perpendicular magnetic field of $B=0.1 \mathrm{~T}$. The magnetic field suppresses ballistic backscattering, since the cyclotron radius $r_{c} \approx 800 \mathrm{~nm}$ is shorter than the smallest feature size of the channel, while the viscous friction stays robust, given that $r_{c}$ is about twice larger than the typical electron-electron scattering length $l_{e e}$. Of course, we cannot completely eliminate all ballistic effects due to crenellations, but the remainder is much smaller as indicated by the data. The light blue circles in Figs. 2(c)-2(e) show the resistance of the crenellated channel at $B=0.1 \mathrm{~T}$. The low-temperature resistance is reduced by the suppression of ballistic effects but is still larger than the straight channel. This result suggests that there is viscous contribution to the resistance of the crenellated channel over a wide temperature range. At higher temperatures, the resistance at $B=0.1 \mathrm{~T}$ approaches the $B=0 \mathrm{~T}$ resistance, as ballistic contributions decline. There is a clear change of slope in the $B=0.1 \mathrm{~T}$ data at $T=10-15 \mathrm{~K}$ which marks a soft transition from ballistic to hydrodynamic transport regimes [23] [see Fig. 10(c) for different choices of $B$ fields]. This is consistent with the crossover temperature expected from the length scales of the system shown in Fig. 2(b).

\section{EXTRACTION OF VISCOSITY AND $l_{e e}$}

\section{A. Extraction of viscosity}

To quantitatively extract the viscosity $\nu$, we use only the $B=0$ experimental data and solve the Navier-Stokes equations with perfect slip (no-stress) boundary conditions (Appendix F) [24-26]: 


$$
\begin{aligned}
\mathbf{v} / \tau_{\mathrm{mfp}}+\mathbf{v} \cdot \nabla \mathbf{v}-\nu \nabla^{2} \mathbf{v} & =-\nabla \Phi / m^{*} \\
\nabla \cdot \mathbf{v} & =0 .
\end{aligned}
$$

Here, $\mathbf{v}$ is the velocity field, $\tau_{\mathrm{mfp}}$ is the mean free time due to phonons and disorder, $m^{*}$ is the effective mass of electron, $\nu$ is the kinematic viscosity, and $\Phi$ is the electrochemical potential. The mean free time is extracted directly from the measured resistance of the straight channels, $R_{\text {straight }}=m^{*} L /\left(n W e^{2} \tau_{\mathrm{mfp}}\right)$. Hence, the viscosity $\nu$ is the only unknown parameter. We choose a particular value of $\nu$ and numerically solve the Navier-Stokes equations at $B=0$ (see Appendixes D and E). The numerical solution of Eq. (1) for a given $\nu$ gives the velocity field, from which we calculate the dissipation and compare it with the measured resistance (also at $B=0$ ). We repeat this procedure until we find the value of $\nu$ that reproduces the experimental resistance of the crenellated segment. This value is the "experimental value" of $\nu$. For an infinitely large device, the relation to the electron-electron scattering length $l_{e e}$ is $\nu=\frac{1}{4} v_{F} l_{e e}$ (see Appendixes F and G 1). However, for a real device, we need to account for ballistic effects.

\section{B. Ballistic effects}

At low temperatures, $l_{e e}$ approaches the critical sample dimensions, the smallest of which is $l_{\text {cren }}$. This finite size effect causes an apparent saturation of $l_{e e}$ as $T \rightarrow 0$, as ballistic effects become relevant. These ballistic effects can be captured by solving the Boltzmann kinetic equation instead of Navier-Stokes, but this calculation is nontrivial. The calculation can be simplified by assuming that the relaxation time is independent of energy [7,27]. However, in our system, this approximation is invalid, since the ratio $T / E_{F}$ can be as high as 0.7 , making brute-force solution of the Boltzmann equation impractical. Instead, we follow the approach in Refs. $[6,28]$ and capture both the finite size and ballistic effects by introducing an effective viscous mean free path $l_{\mathrm{eff}}$ :

$$
\nu=\frac{1}{4} v_{F} l_{\mathrm{eff}}, \quad \frac{1}{l_{\mathrm{eff}}(T)}=\frac{1}{l_{e e}(T)}+\frac{1}{l_{\mathrm{eff}}(T=0)} .
$$

Here, $l_{\text {eff }}$ is completely independent of scattering of electrons from phonons and impurities and captures the ballistic and viscous effects. The cutoff at the ballistic limit $l_{\text {eff }}(T=0) \approx l_{\text {cren }} / 2.5$ is not a fitting parameter but is extracted from the base temperature $T=0.25 \mathrm{~K}$ measurement, which is dominated by ballistic effects. We assume $l_{\text {eff }}(T=0)$ is $T$ independent, although relaxing this assumption varies the extracted $l_{e e}$ by only $10 \%-20 \%$, as discussed in Appendix G 1. The extraction of $l_{e e}$ from the data is rather reliable at $T \geq 20 \mathrm{~K}$, where the ballistic contribution is relatively small. The extracted values of $l_{e e}$ are less reliable at lower temperatures where the ballistic contribution dominates. Nevertheless, if we extend our method down to $T \approx 10 \mathrm{~K}$, the agreement with theory is still good despite the large error bars [see Fig. 3(a)].

We plot the extracted electron-electron scattering lengths with symbols in Fig. 3(a) as a function of the temperature for an electron density of $n=2.45 \times 10^{11} \mathrm{~cm}^{-2}$ (see Fig. 5 for other densities). Both $l_{\text {eff }}$ and $l_{e e}$ increase with decreasing $T$, with $l_{\text {eff }}$ saturating as it approaches half the crenellation length scale. In contrast, $l_{e e}$, an intrinsic property of the electron liquid, diverges as $T \rightarrow 0$.

It is well known that at very low temperatures the electron-electron scattering length scales $l_{e e} \propto 1 / T^{2}$. However, this approximation is valid only at $T \ll 0.1 E_{F}$ [see Fig. 6(b)], and most data in the literature, including ours, are obtained at $T>0.1 E_{F}$. Therefore, we go beyond the low-temperature approximation, as described in Appendix F. The solid and dashed lines show our theoretical calculations of $l_{e e}$ and $l_{\text {eff }}$ using the random phase approximation (RPA) with $l_{\text {eff }}(T=0)$ as the only parameter taken from experiment. The parameter-free calculation of $l_{e e}$ is in remarkably good agreement with the experiment, given the lack of any fitting parameters.

\section{INDEPENDENT VERIFICATION OF $l_{e e}$ THROUGH MAGNETOTRANSPORT}

To independently check the values of the extracted $l_{e e}$, we show in Figs. 3(b) and 3(c) low-field magnetoresistance measurements at the same carrier density. The crenellated channel exhibits a parabolic negative magnetoresistance, shown in Fig. 3(b), due to the magnetic suppression of the viscosity [30-32]

$$
\nu(B)=\nu(0) \frac{B_{*}^{2}}{B_{*}^{2}+B^{2}}, \quad B_{*}=\frac{p_{F}}{2|e| l_{e e}},
$$

where $p_{F}=\hbar \sqrt{2 \pi n}$ is the Fermi momentum. The characteristic magnetic field $B_{*}$ thus provides a direct measurement of the zero-field $l_{e e}$, without solving the Navier-Stokes equations. Using the second part of Eq. (3) and the experimentally determined values of $l_{e e}$ from Fig. 3(a), we find $B_{*} \approx 105$ and $152 \mathrm{mT}$ for $T=20$ and $25 \mathrm{~K}$, respectively. The magnetoresistance calculated from Eq. (3) is shown by dashed lines in Fig. 3(b) and is in excellent agreement with the measurement. In contrast to the crenellated channels, the straight channels have no noticeable dependence on $B$ over the same field range, as shown in Fig. 3(c). This confirms the absence of viscous effects in the straight channels; i.e., the boundaries are smooth.

Furthermore, lower-temperature magnetoresistance data discussed in Appendix $\mathrm{H}$ provide additional confirmation of the ballistic to hydrodynamic crossover at $T=10-15 \mathrm{~K}$. 

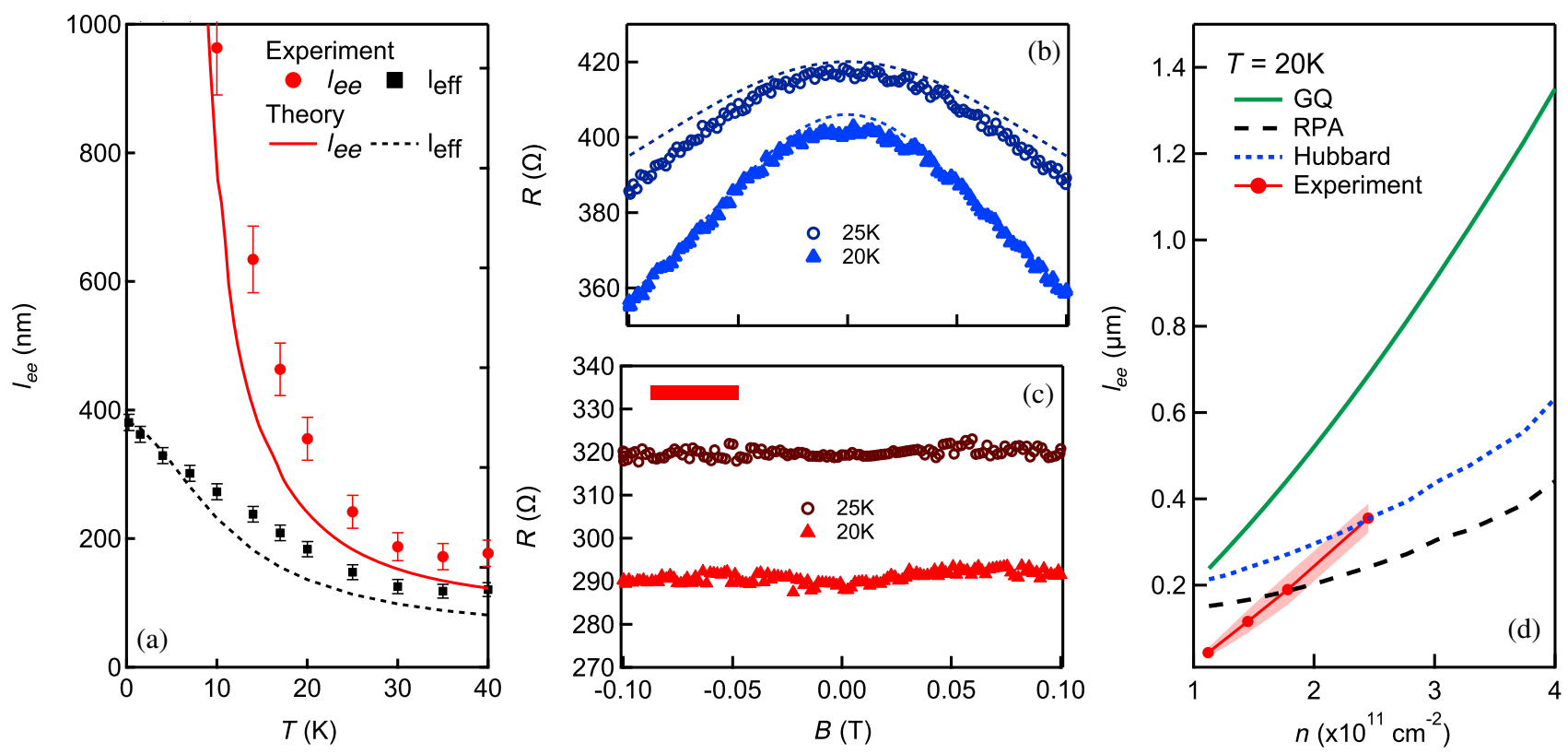

FIG. 3. (a) Electron-electron scattering length $l_{e e}$ and the viscous effective scattering length $l_{\text {eff }}$ as a function of the temperature for an electron density of $n=2.45 \times 10^{11} \mathrm{~cm}^{-2}$. The solid red line is the parameter-free theoretical calculation of $l_{e e}$ for a Fermi liquid using RPA and Boltzmann theory (Appendix F). The dashed black line is the calculated $l_{\text {eff }}$ using Eq. (2). The black squares and red filled circles are the values of $l_{\text {eff }}$ and $l_{e e}$, respectively, extracted from the measured resistance of the crenellated channel. The error bars are due to the uncertainty in the Ohmic component of resistance. (b),(c) High-temperature magnetoresistance of the crenellated and straight channels for $n=2.45 \times 10^{11} \mathrm{~cm}^{-2}$. The straight channel (c) shows no magnetoresistance as expected for smooth sidewalls, whereas the crenellated channel (b) shows a strong negative magnetoresistance due to viscous effects. The dashed lines in (b) are theoretical magnetoresistance curves, calculated using Eq. (3) and the $l_{e e}$ measured in (a). (d) Density dependence of $l_{e e}$ at $T=20 \mathrm{~K}$. Values extracted from the experiment (symbols, with the error margin shown by the shading) are substantially lower than the low $T$ theory of Giuliani and Quinn [29] (as expected) but show a stronger density dependence than predicted theoretically from the more complete RPA (black dashed line) and Hubbard calculations (blue dash-dotted line).

\section{DISCUSSION AND CONCLUSIONS}

Comparing the $l_{e e}$ calculated using the parameter-free theory with the measured values in Fig. 3(a), it is notable that the experimental values are consistently higher than theoretical predictions. This deviation is even more pronounced when examining the variation of $l_{e e}$ (and, hence, the electron quasiparticle lifetime) with carrier density. The $l_{e e}$ calculated in the RPA shows only a weak dependence on $n$, in contrast to the strong dependence measured experimentally in Fig. 3(d). These results suggest that $\tau_{e e}$ has a significant density dependence that is not captured in the RPA. This dependence is especially surprising given the small interaction parameter $r_{s} \sim 1.1-1.5$ of the electron system (see, for example, Ref. [33] and references therein). We have checked that this discrepancy is not an effect of the methods used to solve the Navier-Stokes equations, nor can it be explained by including density-dependent screening effects in the 2DEG. As shown in Appendix G 3, these have a small effect on $l_{e e}$ but do not influence the density dependence of $l_{e e}$. Furthermore, we have gone beyond the RPA, using the Hubbard approximation (Appendixes F and G 2), but the calculated density dependence [blue dashdotted line in Fig. 3(d)] remains inconsistent with experiment. This inconsistency suggests that correlation effects beyond the Hubbard approximation are significant even at relatively low $r_{s}$.

In conclusion, we have created 2D electron channels with perfect slip boundaries, thereby eliminating unknown parameters related to boundary scattering. This method makes it possible to separate extrinsic (phonon and disorder) scattering effects from the intrinsic viscous effects due to electron-electron scattering. From the viscous resistance, we directly extract the electron-electron scattering length. The techniques and analysis introduced here open a new route to probing the finite-temperature quasiparticle lifetime of two-dimensional Fermi liquids over a wide temperature range.

\section{ACKNOWLEDGMENTS}

We acknowledge important discussions with Andre Almeida, Reza Asgari, Gennady V. Stupakov, Joe Wolfe, and Igor Zutic. This work was supported by the Australian Research Council Centre of Excellence in Future LowEnergy Electronics Technologies (CE170100039). D. A. R. acknowledges support from the Engineering and Physical Sciences Research Council, United Kingdom (EP/ 
K004077/1). Devices were made at the New South Wales node of the Australian National Fabrication Facility.

Note added.-Recently, we became aware of a study of viscous behavior in a GaAs 2 DEG using nonlocal measurements [27]. The $l_{e e}$ differ from those obtained here; a comparison of the two approaches is given in Appendix J.

\section{APPENDIX A: CONFIRMATION OF PERFECT SLIP BOUNDARIES AND EXTRACTION OF MEAN FREE TIME $\tau_{\mathrm{mfp}}$}

The solution of fluid equations in a straight channel is well known (see, e.g., Ref. [30]). If the channel is extended in the $x$ direction, the finite slip boundary condition reads $\left.\left(v_{x}-\beta \partial_{y} v_{x}\right)\right|_{\text {boundary }}=0$. For the limit of large slip length $\left(\beta \gg \sqrt{\nu \tau_{\mathrm{mfp}}}\right)$, the channel resistivity is dominated by the usual Ohmic form with higher-order viscous corrections:

$R_{\text {straight }} \rightarrow \frac{L}{W} \frac{m^{*}}{n e^{2} \tau_{\mathrm{mfp}}}\left[1+\frac{2 \nu \tau_{\mathrm{mfp}}}{\beta W}+\mathcal{O}\left(\frac{\nu \tau_{\mathrm{mfp}}}{\beta^{2}}\right)\right]$.

By comparing the experimentally measured resistance in the straight segment to this formula, we can characterize the boundary conditions of our device. We are also able to extract the scattering time $\tau_{\mathrm{mfp}}$ and characterize the scattering impurity and phonon scattering processes. As shown in Fig. 4(a), the experimental data show that the resistance depends only on $L / W$. Shown is the measured resistance in the straight devices with dimensions $W=2.5$ and $5.0 \mu \mathrm{m}$ and $L=25$ and $50 \mu \mathrm{m}$, respectively. By comparing with Eq. (A1), we conclude that for this segment there are no finite slip corrections to the resistivity and the perfect slip (no-stress) boundary condition holds, resulting in uniform fluid flow, as shown in Fig. 1(c) in the main text. In such a uniform flow, the internal viscous friction is irrelevant, because friction plays a role only when different parts of the fluid are in relative motion. Since the effects of the boundary are completely eliminated, the resistance $R_{\text {straight }}$ and corresponding scattering time $\tau_{\mathrm{mfp}}$ are attributed only to impurity and phonon scattering. In principle, the disorder density can slightly vary between different sections of the device. The dotted lines in Fig. 4(a) show the high and low estimates for Ohmic resistance, based on the resistivity of straight sections with two different widths.

\section{APPENDIX B: CONTRIBUTION TO $\tau_{\text {mfp }}$ FROM IMPURITIES}

At zero temperature, the Ohmic resistance is purely due to disorder and, thus, $\tau_{\mathrm{mfp}} \rightarrow \tau_{\text {dis }}$, which we explain with a model distribution of charged impurities in the volume between the gate and the channel. Charged impurities above the channel create a variation in the gate potential. If a positive elementary charge is located above the fluid plane, it creates the electric potential that scatters the electrons. Assuming a volumetric distribution of impurities in the region between the gate and the 2DEG and following Ref. [34], we find approximately that

$$
\begin{aligned}
\frac{1}{\tau_{\mathrm{dis}}} & \approx \frac{\sqrt{2} \pi \hbar n_{i}}{m^{*} \kappa} r_{s}^{3}\left(-\frac{\sqrt{2}}{r_{s}+\sqrt{2}}+\log \frac{r_{s}+\sqrt{2}}{r_{s}}\right), \\
r_{s} & =\kappa /\left(\sqrt{2} k_{F}\right) \sim \sqrt{2} .
\end{aligned}
$$

As shown in Table I, $1<r_{s}<1.5$ has a tight range, and, therefore, we can expand $\tau_{\text {dis }}$ in $n$ to obtain
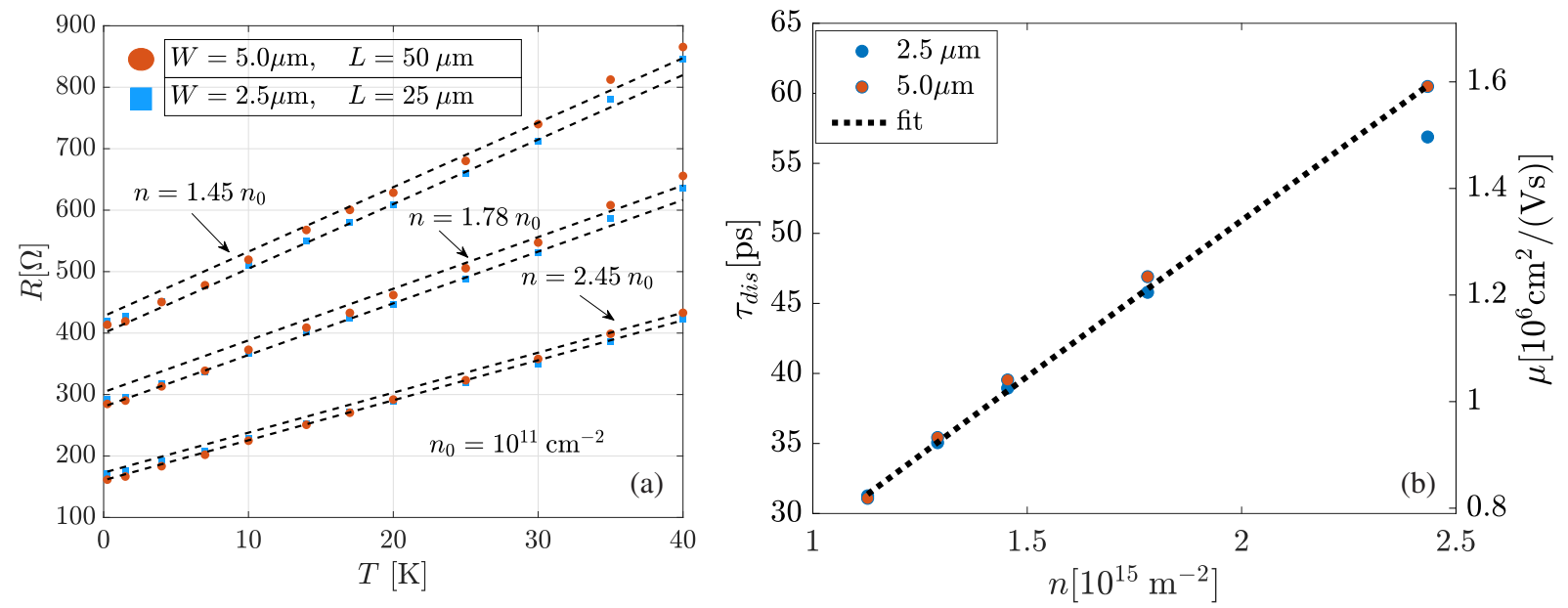

FIG. 4. (a) The resistances in the straight devices with dimensions $W=2.5$ and $5.0 \mu \mathrm{m}$ and $L=25$ and $50 \mu \mathrm{m}$, respectively, as a function of the temperature. The dotted lines are the high and low fits (see Table II). (b) Electron-impurity mean free time and corresponding impurity limited mobility (at $T=0.25 \mathrm{~K}$ ) with respect to number density. A volumetric distribution of $n_{i}=1.13 \times$ $10^{15} \mathrm{~cm}^{-3}$ impurities yields the dotted line based on Eqs. (B1) and (B2) and explains the observed data shown by filled circles. 
TABLE I. Parameters for the resistivity of 2DEG relevant to impurities.

\begin{tabular}{lcll}
\hline \hline Parameter & Expression & Value & \multicolumn{1}{c}{ Description } \\
\hline$\epsilon_{r}$ & & 12.5 & Relative permittivity \\
$\epsilon$ & $\epsilon_{r} 4 \pi \epsilon_{0}$ & $1.39 \times 10^{-9} \mathrm{~F} / \mathrm{m}$ & Permittivity \\
$1 / \kappa$ & $\epsilon \hbar^{2} /\left(2 m^{*} e^{2}\right)$ & $4.94 \mathrm{~nm}$ & Screening length \\
$1 / k_{F}$ & $1 / \sqrt{2 \pi n}$ & $8-10 \mathrm{~nm}$ & Reduced Fermi wavelength $\left(\chi_{F}\right)$ \\
$r_{s}$ & $\kappa /\left(\sqrt{2} k_{F}\right)$ & $1.1-1.5$ & Interaction parameter \\
$z$ & & $\sim 100 \mathrm{~nm}$ & Vertical distance of impurity from 2DEG \\
$n_{i}$ & & $1.13 \times 10^{15} \mathrm{~cm}^{-3}$ & Impurity concentration \\
\hline \hline
\end{tabular}

$$
\begin{aligned}
\tau_{\text {dis }} & \approx \frac{2 m^{*} \kappa}{\pi \hbar n_{i}}\left(0.1+13.9 \frac{n}{\kappa^{2}}+\mathcal{O}\left(n^{3} / \kappa^{6}\right)\right), \\
0.02 & <n / \kappa^{2}<0.06 .
\end{aligned}
$$

A reasonable value of $n_{i} \approx 1.13 \times 10^{15} \mathrm{~cm}^{-3}$ for volumetric density of charged impurities sufficiently accounts for the observed impurity limited scattering lifetime as seen in Fig. 4(b).

\section{APPENDIX C: CONTRIBUTION TO $\tau_{\text {mfp }}$ FROM PHONONS}

The $T$-linear resistance of the straight channels is consistent with deformation potential coupling of electrons to acoustic phonons as outlined below.

From the straight section measurements depicted in Fig. 4(a) and tabulated in Table II, we extract the electron-phonon scattering mean free time from $\rho_{\text {Drude }}-\rho_{\text {dis }}=$ $m^{*} /\left(n e^{2} \tau_{\mathrm{ph}}\right)$ as

$$
\tau_{\mathrm{ph}} \approx 1.55 \mathrm{~ns} \frac{1}{T[\mathrm{~K}]} .
$$

The electron-phonon interaction vertex for phonon coupling is $V_{e-\mathrm{ph}}=\sqrt{\hbar} E_{d} q / \sqrt{2 \rho_{d} \omega_{q}}$, where $E_{d}$ is the deformation potential, $q$ is the wave vector of the phonon, $\rho_{d}$ is the mass density of the crystal, and $\omega_{p}$ is phonon energy. For an acoustic phonon, $\omega_{p}=u q \hbar$, where $u$ is the sound velocity. When an electron scatters from an acoustic phonon, momentum conservation dictates that the phonon

TABLE II. The linear function for the Drude resistivity extracted from the straight geometry. We allow for variation of disorder distribution to explain the small difference between segments with two widths and use these low and high estimates to analyze the Ohmic resistance in the crenellated channel.

\begin{tabular}{lcc}
\hline \hline$\rho_{\text {Drude }}=A_{\mathrm{ph}} T+\rho_{\mathrm{dis}}$ & $A_{\mathrm{ph}}[\Omega / \mathrm{K}]$ & $\rho_{\mathrm{dis}}[\Omega]$ \\
\hline Density $\times 10^{11} \mathrm{~cm}^{-2}$ & & \\
$n=1.45$ & 1.05 & $41.37 \pm 1.38$ \\
$n=1.78$ & 0.84 & $29.22 \pm 1.19$ \\
$n=2.45$ & 0.65 & $16.68 \pm 0.62$ \\
\hline \hline
\end{tabular}

momentum $q \sim p_{F}$, since the electron comes from the vicinity of the Fermi surface. Noting that the Fermi velocity for our choice of density is much larger than the speed of sound in GaAs, $\left(u \sim v_{F} / 100\right)$, the energy of phonon satisfies $\omega_{q} /\left(k_{B} T\right) \sim\left(u / v_{F}\right) E_{F} /\left(k_{B} T\right) \ll 1$, whenever $T \gtrsim 1 \mathrm{~K}$. Therefore, the number of phonons with momentum $q$ can be obtained from the Bose-Einstein distribution as $n_{q}^{b} \sim T / \omega_{q}$. Moreover, since the energy of phonon is small compared to the Fermi energy, the phase space volume of quasielastic scattering is independent of temperature in the range of our experiment. The scattering rate is proportional to the number of phonons, which are populated according to the Bose-Einstein distribution $n^{b}$. Since $n_{q}^{b} \sim k_{B} T / \omega_{q}$, except when $T<1 \mathrm{~K}$, the scattering rate increases linearly with the temperature.

Note that the phonon momentum $q$ is a three-dimensional vector, since phonons are not confined to the quantum well, unlike electrons. This vector results in a form factor of about $F \approx 3 / 2$. Finally, from Fermi's golden rule, we obtain the acoustic phonon limited mobility as

$$
\mu_{\mathrm{ph}}=\frac{2 e \hbar^{3} \rho_{d} u^{2} d}{3 m^{* 2} E_{d}^{2} k_{B} T}
$$

which is consistent with the literature [35-38]. We estimate for GaAs (based on Ref. [35] and references therein) that $\tau_{\mathrm{ph}}$ for a quantum well of thickness $d=10 \mathrm{~nm}$ is $\tau_{\mathrm{ph}} \sim$ $1 \mathrm{~ns} / T[\mathrm{~K}]$ and is very close to our measurements.

Above approximately $40 \mathrm{~K}$, longitudinal optical (LO) polar phonons are activated, and they significantly reduce the mobility in GaAs [38].

\section{APPENDIX D: CONTRIBUTION TO MEASURED RESISTANCE FROM VISCOSITY AND CRENELLATIONS}

Having shown for the straight channel that the boundaries are smooth and flow is uniform [as shown in Fig. 1(c) in the main text], we now describe the role of viscous effects in the crenellated channels [see Figs. 1(d)-1(f) in the main text]. In the crenellated channel, the electron fluid is forced to undergo a sequence of diverging and converging flows. As a result, the velocity of the fluid $\mathbf{v}$ is nonuniform, 
which means that components of the fluid that are in relative motion with respect to each other experience a viscous internal friction force $m^{*} \nu \nabla^{2} \mathbf{v}$. The dissipated power per unit area, due to viscous friction, is then $n m^{*} \nu \mathbf{v} \cdot \nabla^{2} \mathbf{v}$, which we have plotted in Fig. 1(e) in the main text. We integrate the dissipated power over the whole area of the device and, by the conservation of energy, find that the viscous resistance satisfies $R_{\text {viscous }} I^{2}=n m^{*} \nu \int d^{2} x \mathbf{v} \cdot \nabla^{2} \mathbf{v}$. Noting that the current density $\mathbf{J}=|e| n \mathbf{v}$ and the current that runs through the device is $I=J W$, we obtain

$$
R_{\mathrm{viscous}}=\frac{\nu m^{*}}{I^{2} n e^{2}} \frac{1}{2} \int d^{2} x\left(\partial_{i} J_{j}+\partial_{j} J_{i}\right)^{2}=\gamma \rho_{\nu},
$$

where $\rho_{\nu}=\frac{\nu m^{*}}{n e^{2} W^{2}}$.

Numerical solution of the Navier-Stokes equations (NSEs) (see the next section) establishes that $\gamma \sim 60$ for our channel geometry. The value of $\gamma$ is sensitive to how the flow is distributed in the channel and depends on (a) the Reynolds number $\operatorname{Re}=I /(\nu n|e|)$, (b) the relative strength of impurities $\nu \tau_{\mathrm{mfp}} / W^{2}$, and, finally, (c) the geometry, i.e., the ratio of length to width $L / W$ and the crenellation length $l_{\text {cren }} / W$. In Table III, we show the realistic experimental parameters that we use in our simulation. We note that the Reynolds number is small for realistic currents, and it therefore drops out of the problem.
Note that, in addition to viscosity, Ohmic dissipation is also at work in the crenellated channel. The density of power dissipated due to Ohmic resistivity is simply

$R_{\text {Ohmic }} I^{2}=\int d^{2} x \rho_{\text {Drude }} J^{2}, \quad \rho_{\text {Drude }}=\frac{m^{*}}{n e^{2} \tau_{\mathrm{mfp}}}$.

In Fig. 1(d) in the main text, we show the Ohmic power density in the crenellated channel, assuming that the viscosity is zero. Compared to Fig. 1(c) in the main text, it is clear that the density is less, simply because, in the crenellated channel, the effective width is larger. We account for the viscous effects by numerically solving the NSE and explicitly evaluating the integral in Eq. (D2). We determine that the ratio $R_{\text {Ohmic }} / \rho_{\text {Drude }}$ is slightly larger than $L / W=10$, as long as $\sqrt{l_{\mathrm{mfp}} l_{e e}}>0.16 W \approx 400 \mathrm{~nm}$. Above a critical temperature $T_{\text {Ohmic }}^{*}$, the $l_{\text {mfp }}$ shrinks due to frequent collisions with phonons, and, therefore, the Ohmic contribution $R_{\text {Ohmic }}$ drops below the straight segment resistance $L \rho_{\text {Drude }} / W$. Based on the acoustic phonons we analyze in Appendix C, we predict $T_{\text {Ohmic }}^{*}$ to be 48, 90, and $153 \mathrm{~K}$ for lowest, medium, and highest density, respectively. However, we note that, above approximately $40 \mathrm{~K}$, LO phonons significantly reduce the mobility in GaAs [37,38]. Taking this reduction into account, we estimate that the transition to the Ohmic regime will occur at $45-75 \mathrm{~K}$ in the range of densities we consider.

TABLE III. Parameters of electron system organized in four sections. First is Fermi-energy-related scales, controlled by the effective electron mass (GaAs is chosen) and density. Second is the scales related to the $e$ - $e$ and $e$-ph collisions that determine resistivity and viscosity. Third, the geometric and ambient parameters are listed. Finally, the dimensionless numbers of flow are given. Note that the Reynolds number is low, indicating Stokes or "creeping" flow with Ohmic corrections depending on $\sqrt{\nu \tau_{\mathrm{mfp}}} / W$.

\begin{tabular}{|c|c|c|c|}
\hline Parameter & Expression & Value & Description \\
\hline$m^{*}$ & $0.067 m_{e}$ & $6.1 \times 10^{-32} \mathrm{~kg}$ & Effective electron mass \\
\hline$n$ & & $1.45-2.45 \times 10^{11} \mathrm{~cm}^{-2}$ & Electron density \\
\hline$E_{F}$ & $\hbar^{2} \pi n / m^{*}$ & $5.2-8.7 \mathrm{meV}, 60-100 \mathrm{~K}$ & Fermi energy \\
\hline$v_{F}$ & $\sqrt{2 E_{F} / m^{*}}$ & $1.65-2.14 \times 10^{5} \mathrm{~m} / \mathrm{s}$ & Fermi velocity \\
\hline $1 / k_{F}$ & $(2 \pi n)^{-1 / 2}$ & $8.1-10.5 \mathrm{~nm}$ & Reduced Fermi wavelength $\left(\chi_{F}\right)$ \\
\hline$l_{e e}$ & & $>100 \mathrm{~nm}$ & $e-e$ interaction length scale \\
\hline$\tau_{\mathrm{mfp}}$ & & $19.3-60.24 \mathrm{ps}$ & Momentum relaxation time \\
\hline$l_{\mathrm{mfp}}$ & $v_{F} \tau_{\mathrm{mfp}}$ & $3.2-12.8 \mu \mathrm{m}$ & Mean free path \\
\hline$\rho_{\text {Drude }}$ & $\left(n e^{2} \tau / m^{*}\right)^{-1}$ & $20-80 \Omega$ & Ohmic resistivity \\
\hline$\nu$ & $l_{e e} v_{F} / 4$ & $4.1-16.5 \times 10^{-3} \mathrm{~m}^{2} / \mathrm{s}$ & Kinematic viscosity \\
\hline$L$ & & $25 \mu \mathrm{m}$ & Total device length \\
\hline$W$ & & $2.5 \mu \mathrm{m}$ & Width of channel at the entrance \\
\hline$I$ & & $<200 \mathrm{nA}$ & Current through device \\
\hline$v$ & $I /(e n W)$ & $<350 \mathrm{~m} / \mathrm{s}$ & Fluid velocity \\
\hline$T$ & & $20 \mathrm{~K}$ & Ambient temperature \\
\hline & $m^{*} \nu /\left(n e^{2} W^{2}\right)$ & $1.1-4.3 \Omega$ & Viscous resistivity \\
\hline$\Delta T / \Delta V$ & $e /\left(k_{B} T\right)$ & $43.3 \mathrm{~K} / \mathrm{mV}$ & Estimated temperature gradient per voltage \\
\hline $\operatorname{Re}$ & $v W / \nu$ & $<0.2$ & Reynolds number \\
\hline $\mathrm{Gu}$ & $\sqrt{\tau_{\mathrm{mfp}} \nu} / W$ & $0.2-0.9$ & $\begin{array}{l}\text { Gurzhi number: Relative strength } \\
\text { of viscous to Ohmic resistance }\end{array}$ \\
\hline
\end{tabular}



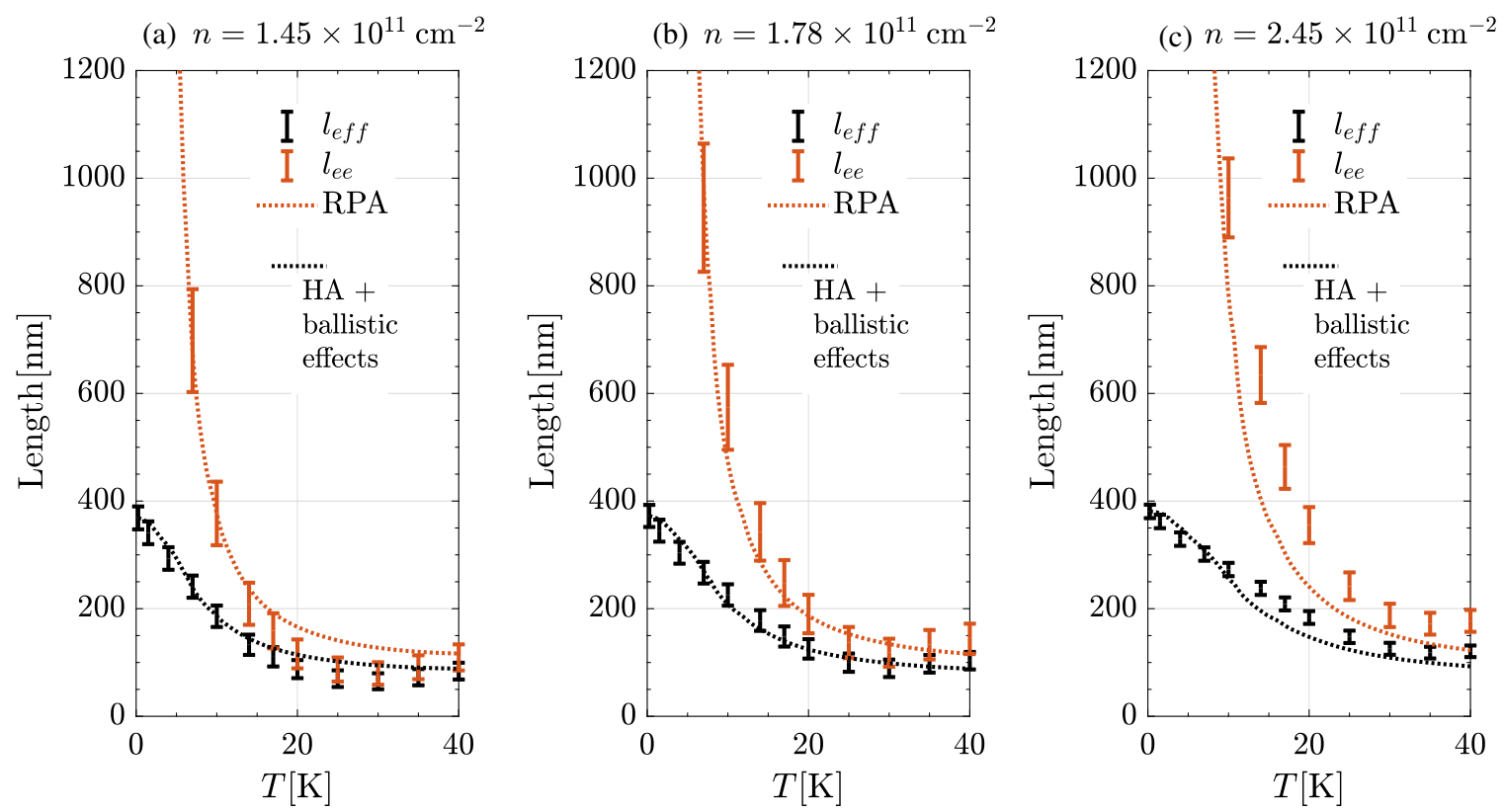

FIG. 5. Extracted $l_{e e}$ and the viscous effective length $l_{\text {eff }}=4 \nu / v_{F}$ as a function of the temperature for three different number densities: (a) 1.45 , (b) 1.78 , and (c) 2.45 in units of $10^{11} \mathrm{~cm}^{-2}$. The error bars are the $l_{e e}$ and the viscous effective length $l_{\text {eff }}=v_{F} /(4 \nu)$ extracted from resistance data by solving Navier-Stokes equation (E1) and using the formula in Eq. (G1) that captures size effects. The uncertainty is due to the possible variation of impurity concentration as discussed in Appendixes A and D. Dotted orange lines are the theoretical calculation of the $l_{e e}$ using RPA and Boltzmann theory, with no free parameters, as outlined in Appendix F. The dotted black line shows the viscous effective length we calculate by using theoretical $l_{e e}$ and Eq. (G1).

Note that, when we measure the resistance of the crenellated channel, the viscous and Ohmic contributions cannot be determined separately. Therefore, in order to deduce the viscosity from the resistance, one needs a prior knowledge of the Ohmic component $\rho_{\text {Drude }}$ or $\tau_{\mathrm{mfp}}$. We use the values we obtain from the straight segment (see Table II). The density of impurities might vary over different channels, and, therefore, the precise value of $\rho_{\text {Drude }}$ in the crenellated channel is unknown. We calculate the error margins on $\rho_{\text {Drude }}$, by using the bounds on the Ohmic resistance in Table II, obtained from the two different straight channels with $W=2.5$ and $5 \mu \mathrm{m}$.

Knowing the density of the fluid, the input current, and the bounds on $\rho_{\text {Drude }}$, we numerically calculate the viscosity that reproduces the measured resistance, as we discuss further below in Appendix E. In Fig. 5, the black error bars show the bounds on viscosity converted to an effective viscous length $l_{\text {eff }}=4 \nu / v_{F}$, as a function of the temperature for three different densities. We then extract $l_{e e}$ by using Eq. (2). Also in Fig. 5, the orange error bars show the bounds on experimentally extracted $l_{e e}$. Note that the error in $l_{e e}$ grows as the temperature is decreased. This result is consistent with Eq. (2), from which we obtain the ratio of error in $l_{e e}$ to $l_{\text {eff }}$ as $\Delta l_{e e} / \Delta l_{\text {eff }}(T) \propto l_{\text {eff }}^{2}(T=0) /\left[l_{\text {eff }}(T=0)-l_{\text {eff }}(T)\right]^{2}$.

Below, we further explain the extraction of $l_{e e}$ from the experiment (i.e., the resistance measurements) and its theoretical calculation.

\section{APPENDIX E: NUMERICAL SOLUTION OF THE NAVIER-STOKES EQUATION}

The number density of electrons is fixed by the top gate electrode and is constant throughout the fluid. Consequently, the steady state flow obeys the incompressible NSEs [24-26,39]

$$
\begin{gathered}
\frac{\mathbf{v}}{\tau_{\mathrm{mfp}}}+\mathbf{v} \cdot \nabla \mathbf{v}-\nu \nabla^{2} \mathbf{v}=-\nabla \Phi / m^{*}, \\
\nabla \cdot \mathbf{v}=0 .
\end{gathered}
$$

Here, $\mathbf{v}$ is the local macroscopic fluid velocity vector following the flow of positive charge per convention, $\nu$ is the kinematic viscosity, $n$ is the $2 \mathrm{D}$ number density, $m^{*}$ is the effective mass of fluid particles, and $-|e|$ is the electron charge. On the left-hand side in Eq. (E1), the first term is due to damping created by impurities and phonons, the second is due to convective acceleration, and, finally, the third represents the viscous force. On the right-hand side, the electrostatic potential gradient appears as a source term that drives the fluid. The electrodes measure $\Phi=|e| V$, which contains both the pressure and electric effects, so that we have $\nabla \Phi=n^{-1} \nabla p+|e| \nabla \phi$.

Once the scattering mean free time $\tau_{\mathrm{mfp}}$ of electrons from impurities and phonons and the viscosity $\nu$ is known, we can solve this equation by imposing the boundary 
conditions, which we explain below. First, we fix the velocity at the input terminal that is $W=2.5 \mu \mathrm{m}$ wide [as shown on the left side in Fig. 1(d) in the main text]. The input velocity field is uniform and points in the $x$ direction, that is, parallel to the surface normal of the input terminal. The magnitude of input velocity is determined by the current as $I=|e| n v_{\text {in }} W$. At the output terminal [shown on the right side in Fig. 1(f) in the main text], we fix the pressure $p=0$ that amounts to saying that it is kept at the reference voltage $V_{\text {out }}=0$.

We assume that the sidewalls of the crenellated channel do not "leak" current (impenetrable) and are perfectly smooth (no-stress or perfect slip). If the tangent and normal vectors at the boundary are denoted by $\hat{t}$ and $\hat{n}$, respectively, impenetrability of the boundary requires that $\left.\hat{n}_{i} v_{i}\right|_{\text {boundary }}=0$ (for no penetration) or $\left.\hat{t}_{i} \sigma_{i j} \hat{n}_{j}\right|_{\text {boundary }}=0$ (for no stress) [39]. We note that the stress tensor of the incompressible fluid is the sum of pressure and viscous stresses $\sigma_{i j}=-p \delta_{i j}+n m^{*} \nu\left(\partial_{i} v_{j}+\partial_{j} v_{i}\right)=-p \delta_{i j}+\sigma_{i j}^{\prime}$. A more general boundary condition for arbitrary slip is derived in Ref. [17], where $\left.\beta \hat{t}_{i}\left(\partial_{i} v_{j}+\partial_{j} v_{i}\right) \hat{n}_{j}\right|_{\text {boundary }}=\left.\hat{t}_{i} v_{i}\right|_{\text {boundary }}$, where $\beta$ is the slip length and, once again, $\hat{t}$ and $\hat{n}$ are tangent and normal vectors, respectively, at the boundary. In the limit $\beta \rightarrow 0$, the no-slip boundary condition is obtained, which applies to most molecular fluids flowing in everyday vessels. In the limit $\beta \rightarrow \infty$, we recover the perfect slip (no-stress) condition that holds in our channel, that we verify by measuring the straight channels with differing widths (see Appendix A).

With the above boundary conditions, we sweep the mean free time $\tau_{\mathrm{mfp}}$ and viscosity $\nu$ and numerically calculate the resistance as $R=V_{\text {in }} / I$, which we tabulate as a function of $\nu$ and $\tau_{\mathrm{mfp}}$. We use this look-up table to extract $\nu$ from the measured resistance, given $\tau_{\mathrm{mfp}}$ that we experimentally obtain from the straight channels. Table III gives a summary of parameters used in the simulations. The solution of NSE gives the current density $\mathbf{J}=n|e| \mathbf{v}$ everywhere in the channel. Then, using Eqs. (D1) and (D2), we calculate, respectively, the viscous and Ohmic components of resistances. We find that the dimensionless ratio $\gamma=$ $R_{\text {viscous }} / \rho_{\nu}$ is a weakly decaying function of the dimensionless momentum diffusion length $\mathrm{Gu}=\sqrt{\nu \tau_{\mathrm{mfp}}} / W$ (we use the name "Gu" after Gurzhi). For a realistic range of parameters, $\gamma=75,70$, and 62 for $\mathrm{Gu}=0.12,0.21$, and 0.3 , respectively, and saturates to $\gamma \approx 61$ for $\mathrm{Gu}>0.4$. This result means that a larger Drude resistance, owing to a smaller $\tau_{\mathrm{mfp}}$, causes more viscous resistance. The dimensionless ratio $R_{\text {Ohmic }} / \rho_{\text {Drude }}$ assumes the values $10,10.3$, 10.5 , and 10.6 for $\mathrm{Gu}=0.12,0.21,0.3$, and 0.4 , respectively. Noting that $R_{\text {Ohmic }} / \rho_{\text {Drude }}$ is precisely 10 for the straight channel and, therefore, at $\nu=0$, must be $<10$ in the crenellated channel, for it is effectively wider, we conclude that the viscosity tends to increase Ohmic resistance.

\section{APPENDIX F: THEORETICAL CALCULATION FOR THE VISCOSITY AND ELECTRON- ELECTRON SCATTERING RATE}

The Coulomb interaction between electrons is screened by the 2DEG itself. We consider three different approximations for Coulomb screening. The simplest is the contact approximation, that serves as a point for comparison. In this approximation, the Coulomb interaction is perfectly screened and, thus, zero, except two electrons coincide at the same space point. Second is the static random phase approximation (RPA), that assumes that the electron-hole pairs that screen the Coulomb field are uncorrelated. Last, we consider the Hubbard approximation (HA), that partly captures the correlation of electron-hole pairs that participate in the screening process [40]. Explicit forms for the Coulomb interaction $U$ are shown below. The interaction parameter is the radius of a disk that on average contains one electron, measured in terms of the effective Bohr radius and turns out to be $r_{s}=\left(\kappa / \sqrt{2} k_{F}\right)$. It is known that in the limit $r_{s} \rightarrow 0$, that is achieved at high densities where $k_{F} \gg \kappa$, the correlation effects are canceled and $U_{\mathrm{HA}} \rightarrow U_{\mathrm{RPA}}$ [33].

In the relaxation time approximation, the total relaxation rate at momentum $\mathbf{k}$ is the sum of the relaxation rate $1 / \tau_{e e}^{p}$ of a particle state, for which $n_{\mathbf{k}}=1$, and relaxation rate $1 / \tau_{e e}^{h}$ of a hole state, for which $n_{\mathbf{k}}=0$, i.e., $\tau_{e e}(k)^{-1}=$ $\tau_{e e}^{p}(k)^{-1}+\tau_{e e}^{h}(k)^{-1}$, where

$$
\begin{aligned}
\frac{1}{\tau_{e e}^{p}(k)}= & \frac{1}{8 \pi m^{*}} \int d^{2} q d^{2} p \delta\left(-\mathbf{p} \cdot \mathbf{q}+q^{2}\right) n_{\mathbf{p}+\mathbf{k}} \\
& \times\left(1-n_{\mathbf{p}+\mathbf{k}-\mathbf{q}}\right)\left(1-n_{\mathbf{k}+\mathbf{q}}\right)\left|U(q) m^{*} / \pi\right|^{2},
\end{aligned}
$$

and we obtain the $\tau_{e e}^{h}$ after replacing the distribution functions $n \rightarrow 1-n$ in the expression for $\tau_{e e}^{p}$. Figure 6(a) shows the scattering probability at the Fermi surface, $P\left(\epsilon_{k}=E_{F}\right)$, as a function of normalized temperature $T / T_{F}$ for different values of interaction parameter $r_{s}=$ $\kappa /\left(\sqrt{2} k_{F}\right)$ in the three different approximations. We note that for graphene (see, e.g., Refs. [6,41]), the density $n \sim$ $10^{12} 1 / \mathrm{cm}^{2}$ is about 10 times larger than in our case. Moreover, in monolayer graphene, the Fermi velocity is about 10 times larger than our case; using $E_{F}=\hbar v_{F} \sqrt{\pi n}$, we find that $T_{F} \sim 1500 \mathrm{~K}$ that is, about 20 times larger than our sample, where $T_{F} \sim 60-100 \mathrm{~K}$ (see Table III). Therefore, unlike graphene samples where the Fermi liquid theory estimation for a degenerate gas $l_{e e} \sim 1 / T^{2}$ holds to a large extent, the electrons in our GaAs samples go beyond this estimate and satisfy $\tau \sim 1 / T$ even at relatively low temperatures $T \sim 6-10 \mathrm{~K}$ [which corresponds to $T / T_{F} \sim 0.1$; see Fig. 6(a)].

When the electronic distribution function is disturbed from local thermodynamic equilibrium, ee scattering 
(a)
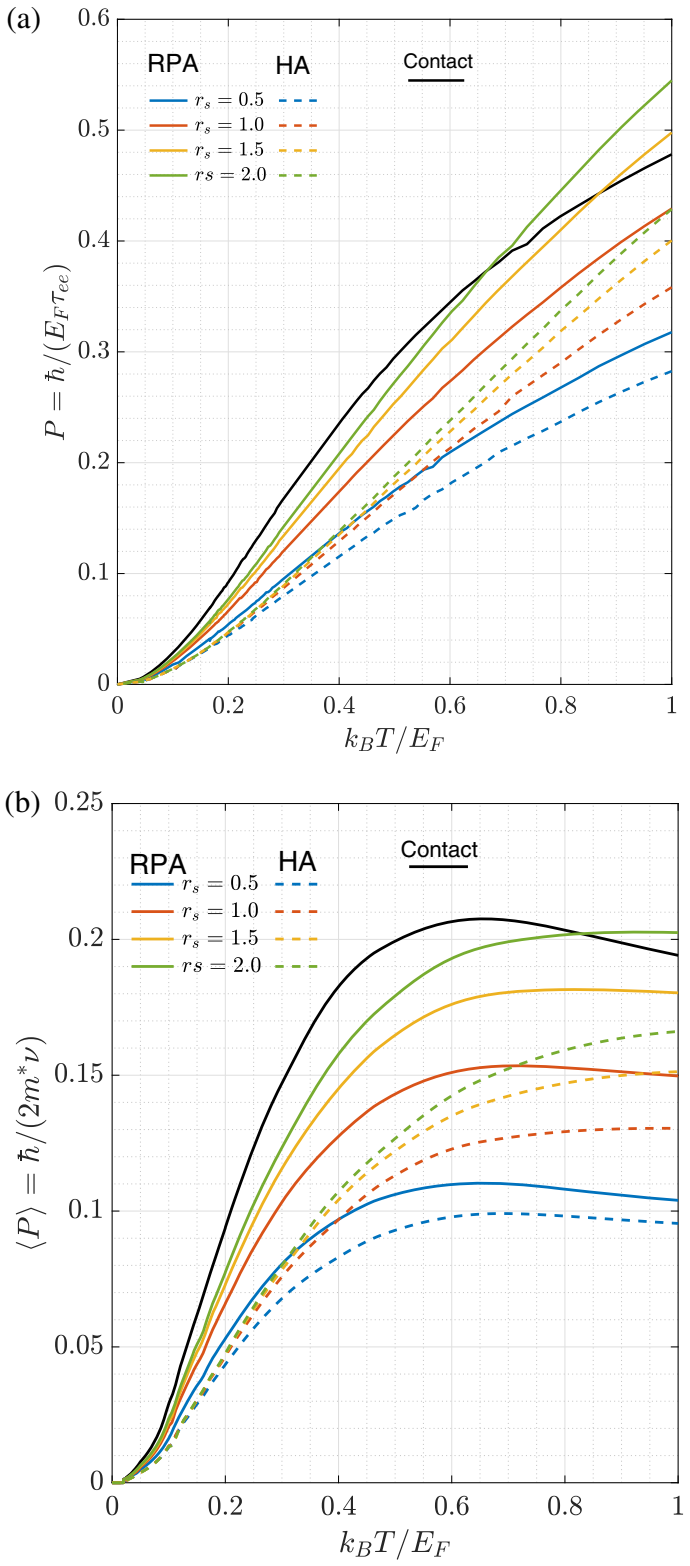

FIG. 6. (a) The scattering probability at the Fermi surface, $P\left(\epsilon_{k}=E_{F}\right)$, and (b) the dimensionless inverse viscosity $\langle P\rangle=\hbar /\left(2 m^{*} \nu\right)$, and, equivalently, $\langle P\rangle=2 /\left(k_{F} l_{e e}\right)$, as a function of normalized temperature $T / T_{F}$ for different values of interaction parameter $r_{s}=\kappa /\left(\sqrt{2} k_{F}\right)$ in the three different approximations in Eq. (G2), namely, contact interaction, RPA, and HA. As $r_{s}$ decreases, the trend RPA $\rightarrow$ HA is clearly visible. At low temperatures, only the Fermi surface contributes to the average; hence, $\langle P\rangle \rightarrow P$ or $l_{e e} \rightarrow v_{F} \tau_{e e}$.

provides the relaxation mechanism that restores the equilibrium. Therefore, we can calculate viscosity by appropriately expanding the kinetic equation around the local thermodynamic equilibrium [42]. This derivation is formally equivalent to the linear response theory of viscous transport [43]. The length scale associated with viscous relaxation differs from the naive interaction length $v_{F} \tau_{e e}$, especially when the distribution function broadens due to high temperatures. This distinction is recognized in the literature (see, for example, Ref. [6]).

Solving the kinetic equation for a small deviation from thermal equilibrium, we can find that the shear stress is proportional to the rate of strain [44]. Here, the proportionality constant is viscosity. For the transport mean free path, or simply ee length in an infinite system, we get

$$
l_{e e}=\frac{4 \nu}{v_{F}}=\frac{v_{F}}{E_{F}^{2}} \int d \epsilon \epsilon^{2} \tau_{e e}(\epsilon)\left(-\partial_{\epsilon} n\right),
$$

which agrees with the microscopic formula for viscosity in the literature (see, for example, Ref. [42]). Figure 6(b) shows the averaged scattering probability, or inverse normalized $l_{e e},\langle P\rangle=2 /\left(k_{F} l_{e e}\right)$ as a function of normalized temperature $T / T_{F}$ for different values of interaction parameter $r_{s}=\kappa /\left(\sqrt{2} k_{F}\right)$ in the three different approximations in Eq. (G2). To reiterate, $l_{e e}$ denotes the mean free path associated with viscous transport rather than the naive length $v_{F} \tau_{e e}$. Note, however, that at low $T$, since the distribution function is peaked at the Fermi surface, the distinction disappears: $l_{e e} \rightarrow v_{F} \tau_{e e}$.

\section{APPENDIX G: ADDITIONAL CONSIDERATIONS}

\section{Relation of viscosity to $l_{e e}$ in the quasiballistic regime}

The viscous transport length scale receives ballistic corrections, when $l_{e e}$ becomes comparable to the size of the system. Here, we present a physical explanation. Hydrodynamics apply when local thermodynamic equilibrium is established. The size of the region in which electrons can thermalize and form a joint local velocity is $l_{e e}$. The viscous power dissipation is not proportional to local current density, but it is derivative, as we show in this Appendix [Eqs. (D1)]. Therefore, dissipation is not local, unlike the dissipation due to $\tau_{\mathrm{mfp}}$, which is always local. When $l_{e e}$ grows comparable to the size, there is a smooth crossover from hydrodynamics, defined in a small volume of size $l_{e e}$ to completely nonlocal, ballistic transport. (The crossover regime is analyzed, for example, in Ref. [42].) The cutoff occurs when $l_{e e}$ becomes comparable to the size.

Following Refs. $[6,28]$, which show that the ballistic and hydroconductance are additive, we capture both the finite size and ballistic effects by introducing an effective viscous length $l_{\text {eff }}=4 \nu / v_{F}$ :

$\nu=\frac{1}{4} v_{F} l_{\mathrm{eff}}, \quad \frac{1}{l_{\mathrm{eff}}(T)}=\frac{1}{l_{e e}(T)}+\frac{1}{l_{\mathrm{eff}}(T=0)}$,

where the cutoff at the ballistic limit $l_{\text {eff }}(T=0) \approx l_{\text {cren }} / 2.5$ is not a fitting parameter but is extracted from the base temperature $T=0.25 \mathrm{~K}$ measurement, which is dominated by ballistic effects.

In our analysis, we assume $L_{b}=l_{\text {eff }}(T=0)$ is $T$ independent. However, even if $L_{b}$ varies with temperature 


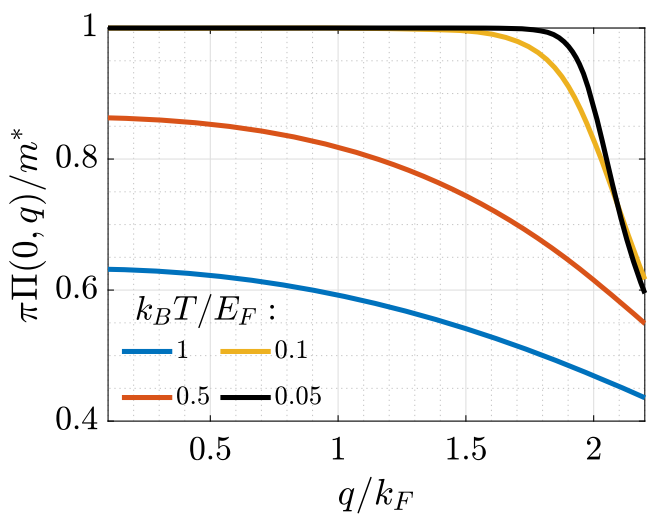

FIG. 7. Normalized polarization function with respect to momentum $q$, for different temperatures.

and changes by $100 \%$, it gives only $10 \%-20 \%$ correction to our values of $l_{e e}$ at $T>20 \mathrm{~K}$. This result is practically within the error bars. Of course, the values of $l_{e e}$ at $T=$ $10 \mathrm{~K}$ are more sensitive to $L_{b}$. However, the agreement of the measured $l_{e e}(T=10 \mathrm{~K})$ with the theory indicates that $L_{b}(T=10 \mathrm{~K}) \approx L_{b}(T=0.25 \mathrm{~K})$.

We present both the viscous effective length and $l_{e e}$ in Fig. 5, for three different densities, and compare it to the theoretical estimates based on RPA. The black error bars are bounds on viscous effective length explained in Appendix D. The orange error bars represent the true $e e$ length $l_{e e}$ that is obtained using the bounds on $l_{\text {eff }}$ and the formula Eq. (G1), that subtracts the ballistic contributions. The dotted orange lines are the RPA prediction. By combining the theoretical $l_{e e}$ taken from Fig. 6(b) in Appendix F with the formula Eq. (G1) using the experimentally obtained ballistic cutoff $l_{\text {eff }}(T=0) \approx l_{\text {cren }} / 2.5$, we arrive at the dotted black curves, that represent the theoretical predictions for viscous effective length $l_{\text {eff }}$.

\section{Hubbard approximation versus the random phase approximation}

The interaction potential in these three approximations read (we set $\hbar=1$ wherever convenient)

$$
U=\left\{\begin{array}{l}
U_{\text {contact }}=\pi / m^{*}, \\
U_{\mathrm{RPA}}=\frac{U_{0}}{1+U_{0} \Pi_{0}^{R}}, \\
U_{\mathrm{HA}}=\frac{U_{0}\left(1-U_{0} G \Pi\right)}{1+U \Pi(1-G)},
\end{array}\right.
$$

where $\Pi_{0}$ is the polarization operator and $U_{0}=\left(\pi \kappa / m^{*} q\right)$ is the bare Coulomb potential with $\kappa^{-1}=\left(\epsilon / 2 m^{*} e^{2}\right)$ as the inverse screening length.
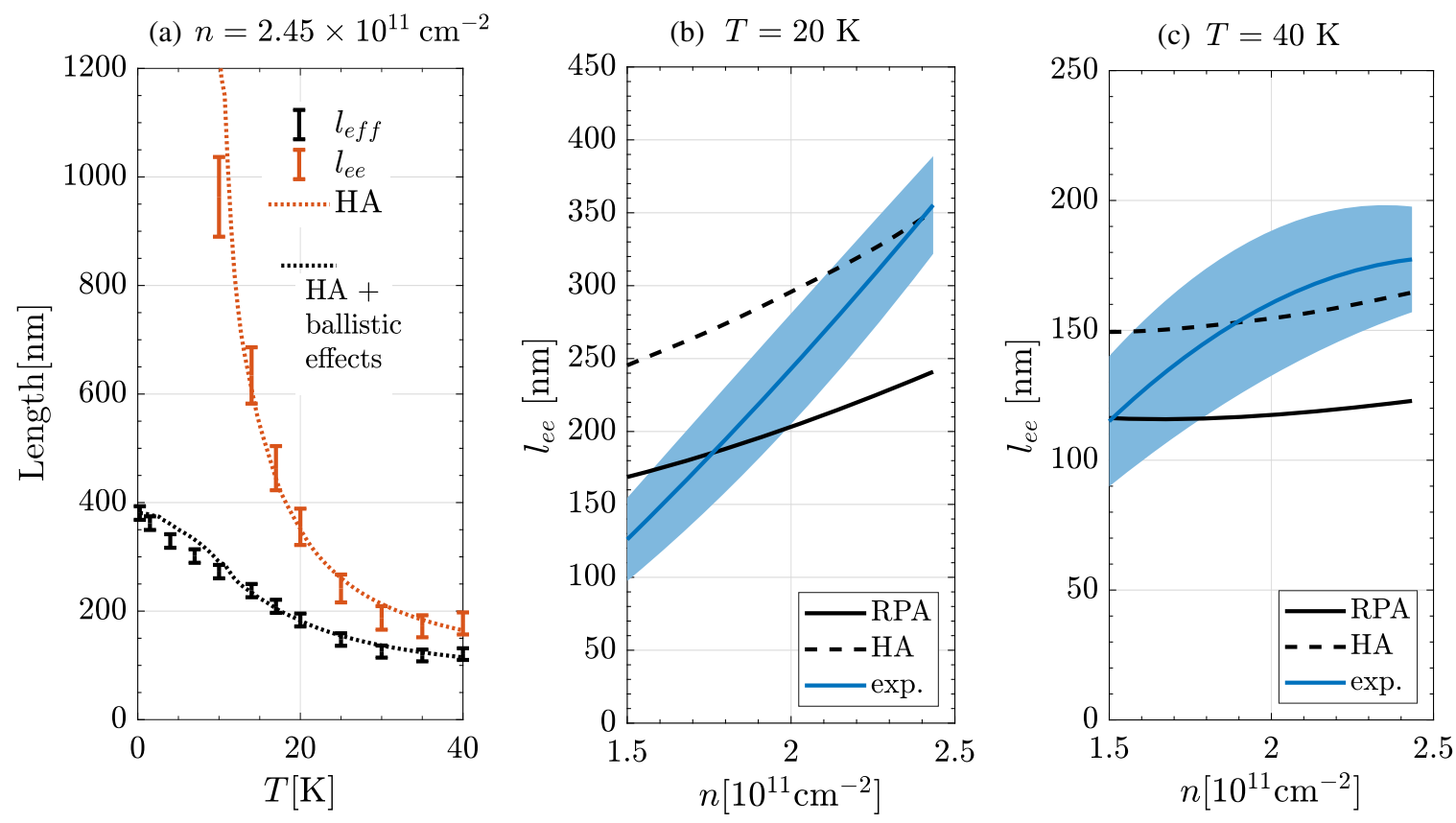

FIG. 8. (a) The measured $l_{e e}$ shown by error bars, compared to the theory with the Hubbard local field correction (dotted orange lines) described in Appendix $\mathrm{F}$ and the measured effective length $l_{\text {eff }}$ that contains low- $T$ ballistic corrections, compared to the theory with the Hubbard local field correction (dotted black lines). See similar plots in Fig. 3, where the dotted theory curves are based on RPA. The density dependence of $l_{e e}$ at (b) $T=20 \mathrm{~K}$ and (c) $T=40 \mathrm{~K}$ in the Hubbard and RPA approximations compared to the experimentally measured values shown by the blue solid line. The shaded area depicts the uncertainty due to the possible variation of impurity concentration as discussed in Appendixes A and D. The correlation effects captured by HA but missed in RPA account for an approximately $50 \%$ increase in the interaction length yet do not explain the strong density dependence observed in the experimental data. 
In Fig. 7, the normalized polarization function $\Pi(0, q) / \Pi(0,0)=\Pi(0, q) \pi / m^{*}$ is given as a function of the temperature and momentum $q$.

The temperature-dependent local field correction $G$ is given as

$$
G(q, T)=\frac{1}{2} \frac{q}{\sqrt{q^{2}+2 m^{*} \mu(T)+\kappa^{2}}} .
$$

As seen in Fig. 5, going from low to high number density, the midpoints of the error bars that represent the experimentally obtained $l_{e e}$ rise faster than the dotted lines that represent the RPA prediction. The failure of the RPA indicates the strong correlation effects. Indeed, as seen in Fig. 8, the prediction based on Hubbard approximation is about 50\% higher than the RPA prediction; hence, the correlation effects are strong despite the relatively low interaction parameter $r_{s}<2$.

\section{3. "Boundary rounding" cannot explain the strong observed density dependence of $l_{e e}$}

One might argue that the strong dependence of the experimentally obtained $l_{e e}$ on $n$ might be due to the variation of 2DEG boundary with particle density.

In the previous analysis, we assume that the shape of the electron liquid repeats the shape of the metallic gate with sharp corners, Fig. 1(a) in the main text. To be more realistic, since the 2DEG is approximately $150 \mathrm{~nm}$ below the gate, the physical shape of the boundary is somewhat
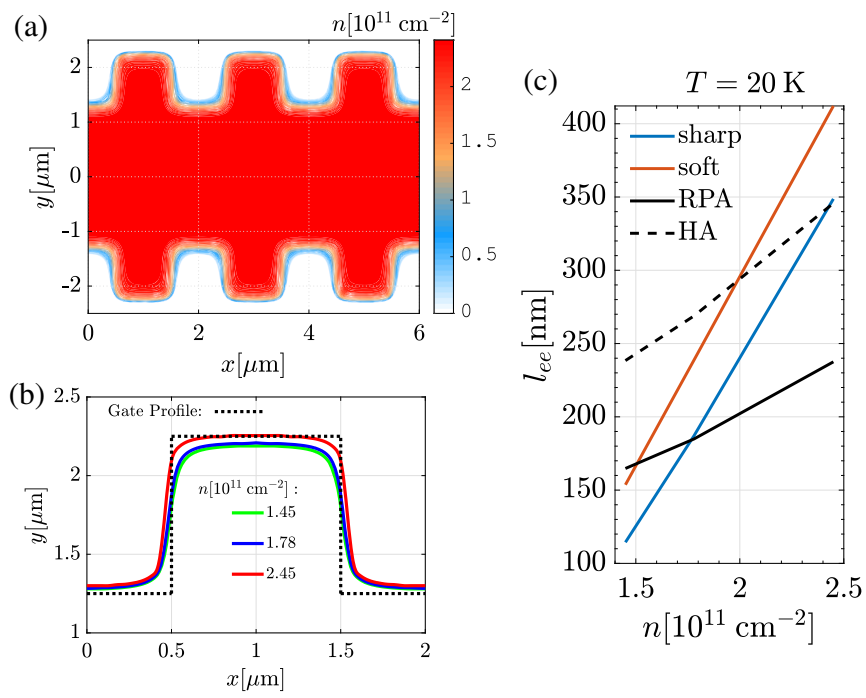

FIG. 9. (a) The charge contour plot over the plane of 2DEG for the highest gate voltage $V=1.6 \mathrm{~V}$, where the number density deep inside the channel (along $y=0$ ) is $n=2.45 \times 10^{11} \mathrm{~cm}^{-2}$. (b) The electrical shape of the channel near the crenellation for three different densities, compared to the top gate marked by the dotted black line. The boundary of the channel is assumed to be the contour on which the density is half its maximum value. (c) The comparison of $l_{e e}$ at $T=20 \mathrm{~K}$ extracted by assuming boundaries of the top gate and the softened boundaries in (a). rounded. To find the actual shape, in this subsection, we solve Thomas-Fermi-Poisson equations (TFPEs) for the system $2 \mathrm{DEG}+$ gate. Solution of TFPEs shows that the electron density in the center of 2DEG channel depends of the gate voltage in the following way:

$$
n\left[10^{11} \mathrm{~cm}^{-2}\right]=3.2649 \times V_{g}[\mathrm{~V}]-2.7901 .
$$

The three densities we measure $(1.45,1.78$, and $2.45 \times 10^{11} \mathrm{~cm}^{-2}$ ) correspond to the voltages $1.3,1.4$, and $1.6 \mathrm{~V}$, respectively. In Fig. 9(a), we present a color map of the charge density plot for $n_{c}=2.45 \times 10^{11} \mathrm{~cm}^{-2}$. Of course, density varies smoothly from $n_{c}$ down to zero. We define the effective boundary of the electron liquid as a line where density is equal to $n_{c} / 2$. Effective boundaries are shown in Fig. 9(b) for three values of $n_{c}$. Having the rounded boundaries, we solve again NSE and, hence, find the values of $l_{e e}$ that are consistent with the measured resistances. This result is presented in Fig. 9(c). We see that, while the rounding of the boundaries slightly changes $l_{e e}$, it does not influence our conclusion about the density dependence.

\section{APPENDIX H: MAGNETORESISTANCE OVER THE ENTIRE RANGE OF TEMPERATURE}

Here, we discuss the magnetoresistance data as further evidence for a transition from the ballistic to fully hydrodynamic regime.

In Figs. 10(a) and 10(b), we show our magnetoresistance data for the crenellated and for the straight segment, respectively. Here, we plot $R-R_{\text {straight }}(B=0)$, because the straight segment resistance is practically $B$ independent (apart from Shubnikov-de Haas quantum oscillations) and, therefore, $R_{\text {straight }}(B=0)$ gives a natural zero level. The data for the broader range of magnetic field are taken only at $T=0.25 \mathrm{~K}$. For the crenellated segment, the strongly $T$-dependent parabolic negative magnetoresistance at higher $T$ is a consequence of the viscous flow and boundary scattering in the hydrodynamic regime. In addition, the $T=0.25 \mathrm{~K}$ curve of the crenellated sample shows a double hump structure related to the interplay between $W$ and $r_{c}$ in the presence of effectively diffusive scattering from the crenellated walls. This structure is a well-understood signature of ballistic transport [21]. At larger fields, the $T=0.25 \mathrm{~K}$ curve shows Shubnikov-de Haas oscillations. Therefore, we limit our range to $|B|<0.1 \mathrm{~T}$, which allows us to trace the double hump as a function of the temperature. We note that the double hump structure goes away at $T=17-20 \mathrm{~K}$, signifying the onset of the hydrodynamic regime. The data indicate that, even at $T=14 \mathrm{~K}$, the fraction of electrons which propagate ballistically across the channel is very small. Since we already extracted the $l_{e e}$ from the $B=0$ data, we need only the curvature at $B=0$ for a second confirmation of our results through magnetoresistance. Hence, the range $|B|<0.1 \mathrm{~T}$ is more than 

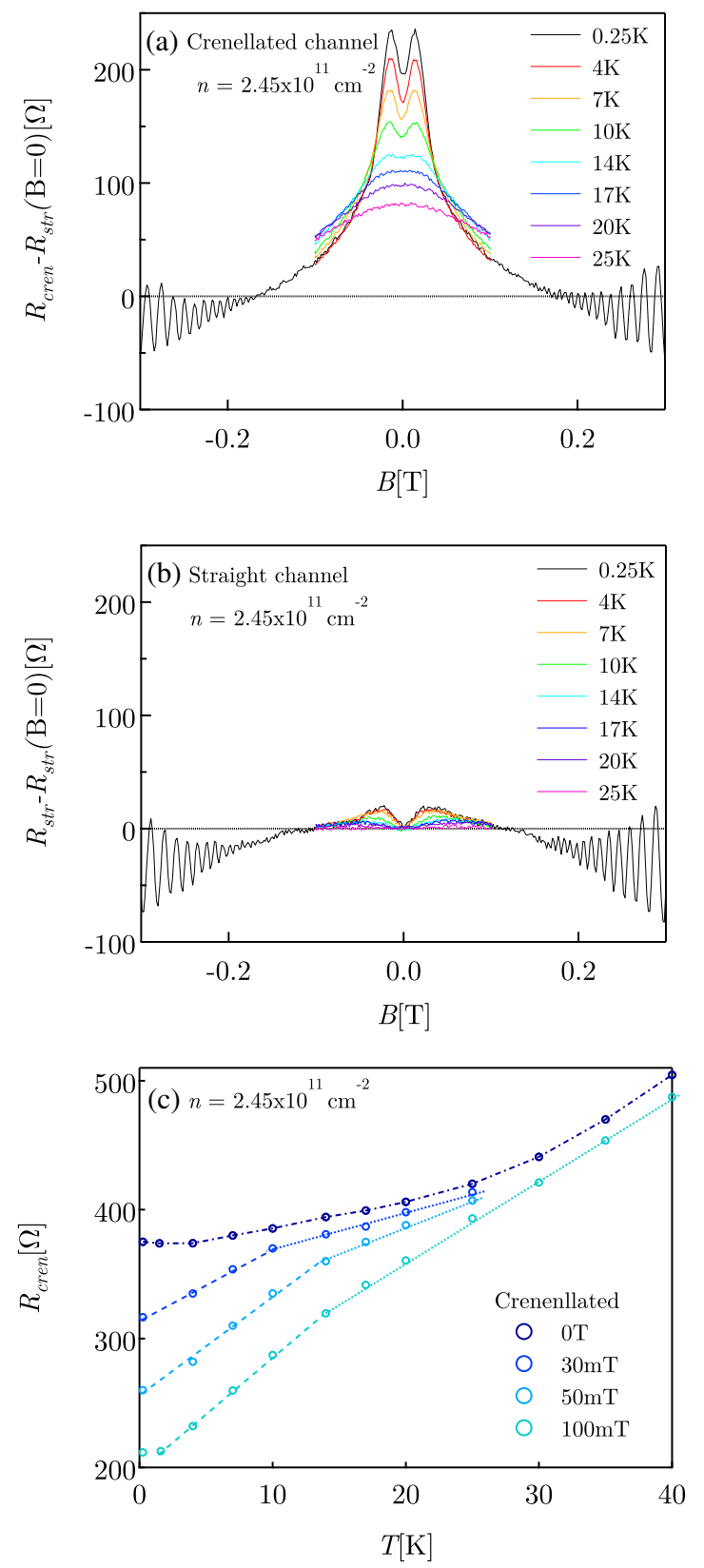

FIG. 10. Magnetoresistance of (a) the crenellated channel after removing the zero-field resistance of the straight channel $R_{\text {straight }}(B=0)$ and (b) the straight channel after removing $R_{\text {straight }}(B=0)$ at $n=2.45 \times 10^{11} \mathrm{~cm}^{-2}$ for different temperatures. (c) Resistance of the crenellated channel as a function of the temperature at different magnetic fields. Dashed (dotted) lines are straight line guides for low (high) temperatures depicting the change in slope.

sufficient to determine this curvature. At larger $B$ fields, the curves are expected to smoothly approach zero.

If we now look at the data from the straight channel, shown on the right in this figure, it does not show the strongly $T$-dependent negative magnetoresistance. At low $T$, a weak double hump structure is visible, which indicates that the electron scattering from the walls is not perfectly specular. However, the effective slip length is longer than the dimensions of the channel, indicated by the resistance of the straight segments presented in Fig. 2, which are practically identical, consistent with vanishing viscous friction in smooth channels.

Of course, the disappearance of the double hump structure at $T=17-20 \mathrm{~K}$ does not imply that the ballistic effects are completely absent. The effects related to the ballistic propagation inside the crenellation still survive, which is why we use the parallel resistance formula to extract $l_{e e}$ at $B=0$. We believe that the quantitative analysis at $B=0$ is much more reliable than that based on the negative magnetoresistance. The magnetoresistance is just a semiquantitative confirmation of the $B=0$ analysis. It is also worth noting that the extraction of $l_{e e}$ from the data is rather reliable at $T \geq 20 \mathrm{~K}$, where the ballistic contribution is relatively small. The extracted values of $l_{e e}$ are less reliable at lower temperatures where the ballistic contribution dominates. Nevertheless, if we extend our method down to $T \approx 10 \mathrm{~K}$, the agreement with theory is still good despite the large error bars.

In Fig. 10(c), we plot the resistance of the crenellated channel as a function of the temperature at $B=0,30,50$, and $100 \mathrm{mT}$. In the low-temperature limit, as the magnetic field $(0.03 \mathrm{~T} \leq B \leq 0.1 \mathrm{~T})$ increases, the ballistic contribution to the resistance of the crenellated is suppressed and $R_{\text {cren }}$ decreases. On the other hand, the magnetic field also reduces the viscous contribution, which accounts for the reduction of $R_{\text {cren }}$ in the high-temperature limit, but at a much slower rate. This different $B$ dependence between the ballistic and viscous resistance results in a clear change of slope of $R_{\text {cren }}(B \neq 0)$ in the range $10-15 \mathrm{~K}$. This change of slope marks the soft transition from ballistic to hydrodynamic transport regimes, which agrees with the crossover temperature expected from the length scales of the system in Fig. 2(b).

\section{APPENDIX I: EXPERIMENTAL METHODS}

The device is fabricated on an undoped GaAs/ $\mathrm{Al}_{x} \mathrm{Ga}_{1-x} \mathrm{As}$ heterostructure comprising a $10 \mathrm{~nm}$ GaAs cap and a $150 \mathrm{~nm}$ AlGaAs layer on a GaAs buffer layer grown by molecular beam epitaxy. Thermally evaporated $\mathrm{Ti} / \mathrm{Au}$ gates are patterned by electron-beam lithography for the active channel region and by standard UV photolithography elsewhere. $N$-type electrical contacts (AuGe) to the heterostructure are thermally evaporated and annealed. $15 \mathrm{~nm}$ of atomic layer deposited $\mathrm{AlO}_{x}$ is used as the dielectric between the contacts and the top gate. The device is measured in a ${ }^{3} \mathrm{He}$ cryostat with a variable temperature range from 0.25 to $40 \mathrm{~K}$. At each temperature, the resistances of the straight and the crenellated channels are measured simultaneously using standard lock-in techniques with a small ac excitation voltage of $100 \mu \mathrm{V}$. The 2DEG density is extracted from the slope of the low-field Hall resistance of the same device at different gate biases. 
(a) $T=13 \mathrm{~K}$

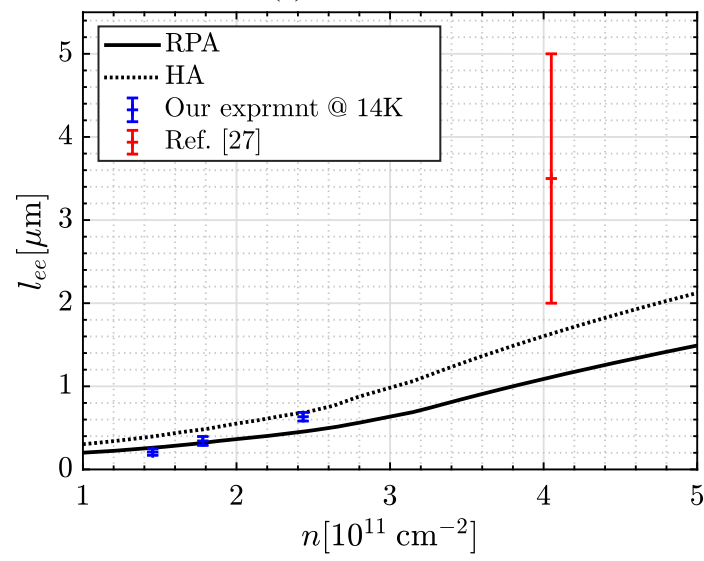

(b) $T=28 \mathrm{~K}$

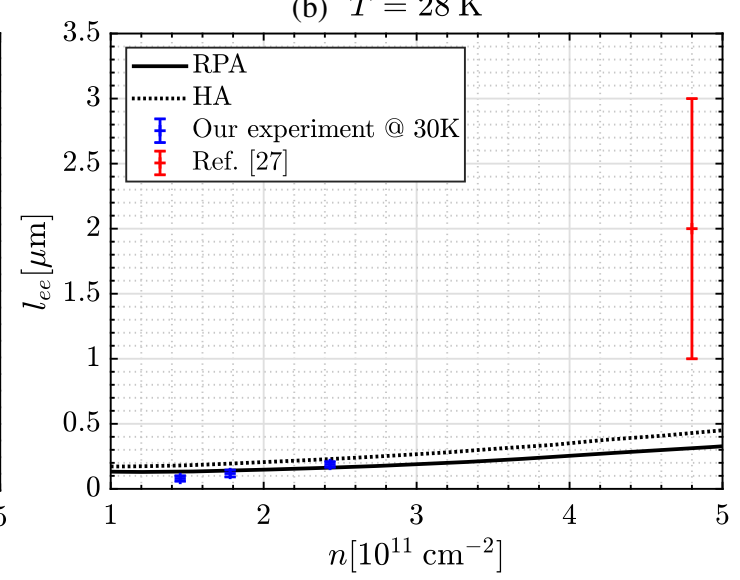

FIG. 11. Comparison of our $l_{e e}$ measurements as a function of electron number density $n$ with that of the recent experiment by Gupta et al. [27] and the parameter-free calculations of $l_{e e}$ as described in Appendix F (a) at $T=13 \mathrm{~K}$ (our data are obtained at $T=14 \mathrm{~K}$ ) and (b) $T=28 \mathrm{~K}$ (our data are obtained at $T=30 \mathrm{~K}$ ).

\section{APPENDIX J: COMPARISON OF $l_{e e}$ MEASUREMENTS FROM PERFECT SLIP DEVICES AND RECENT NONLOCAL MEASUREMENTS}

Here, we compare our results for $l_{e e}$ at zero magnetic field with Ref. [27], which uses elegant nonlocal resistance measurements to detect viscous behavior from vortex formation in devices with multiple point contacts. In Ref. [27], a temperature-dependent calibration parameter is used to extract $l_{e e}$ from comparisons of the measured nonlocal voltages with numerical calculations at two temperatures, 13 and $28 \mathrm{~K}$. Figure 11 compares the two approaches to measuring $l_{e e}$. The nonlocal measurements are more challenging to perform and analyze than the local measurements in the perfect slip devices studied here, with error bars that are about 50 times larger. The extremely high resolution and parameter-free nature of our new technique allows accurate comparison with different theories for $l_{e e}$, bringing the field of electron hydrodynamics to a qualitatively new level.

[1] R. N. Gurzhi, Hydrodynamic Effects in Solids at Low Temperature, Sov. Phys. Usp. 11, 255 (1968).

[2] M. Polini and A. K. Geim, Viscous Electron Fluids, Phys. Today 73, No. 6, 28 (2020).

[3] J. A. Sulpizio, L. Ella, A. Rozen, J. Birkbeck, D. J. Perello, D. Dutta, M. Ben-Shalom, T. Taniguchi, K. Watanabe, T. Holder, R. Queiroz, A. Principi, A. Stern, T. Scaffidi, A. K. Geim, and S. Ilani, Visualizing Poiseuille Flow of Hydrodynamic Electrons, Nature (London) 576, 75 (2019).

[4] M. J. H. Ku, T. X. Zhou, Q. Li, Y. J. Shin, J. K. Shi, C. Burch, L. E. Anderson, A. T. Pierce, Y. Xie, A. Hamo, U. Vool, H. Zhang, F. Casola, T. Taniguchi, K. Watanabe, M. M. Fogler, P. Kim, A. Yacoby, and R. L. Walsworth,
Imaging Viscous Flow of the Dirac Fluid in Graphene, Nature (London) 583, 537 (2020).

[5] D. A. Bandurin, I. Torre, R. K. Kumar, M. B. Shalom, A. Tomadin, A. Principi, G. H. Auton, E. Khestanova, K. S. Novoselov, I. V. Grigorieva, L. A. Ponomarenko, A. K. Geim, and M. Polini, Negative Local Resistance Caused by Viscous Electron Backflow in Graphene, Science 351, 1055 (2016).

[6] R. K. Kumar, D. A. Bandurin, F. M. D. Pellegrino, Y. Cao, A. Principi, H. Guo, G. H. Auton, M. B. Shalom, L. A. Ponomarenko, G. Falkovich, K. Watanabe, T. Taniguchi, I. V. Grigorieva, L. S. Levitov, M. Polini, and A. K. Geim, Superballistic Flow of Viscous Electron Fluid through Graphene Constrictions, Nat. Phys. 13, 1182 (2017).

[7] D. A. Bandurin, A. V. Shytov, L. S. Levitov, R. K. Kumar, A. I. Berdyugin, M. Ben Shalom, I. V. Grigorieva, A. K. Geim, and G. Falkovich, Fluidity Onset in Graphene, Nat. Commun. 9, 4533 (2018).

[8] J. Gooth, F. Menges, N. Kumar, and V. Süß, C. Shekhar, Y. Sun, U. Drechsler, R. Zierold, C. Felser, and B. Gotsmann, Thermal and Electrical Signatures of a Hydrodynamic Electron Fluid in Tungsten Diphosphide, Nat. Commun. 9, 4093 (2018).

[9] J. Crossno, J. K. Shi, K. Wang, X. Liu, A. Harzheim, A. Lucas, S. Sachdev, P. Kim, T. Taniguchi, K. Watanabe, T. A. Ohki, and K. C. Fong, Observation of the Dirac Fluid and the Breakdown of the Wiedemann-Franz Law in Graphene, Science 351, 1058 (2016).

[10] P. J. W. Moll, P. Kushwaha, N. Nandi, B. Schmidt, and A. P. Mackenzie, Evidence for Hydrodynamic Electron Flow in $\mathrm{PdCoO}_{2}$, Science 351, 1061 (2016).

[11] G. M. Gusev, A. S. Jaroshevich, A. D. Levin, Z. D. Kvon, and A. K. Bakarov, Stokes Flow around an Obstacle in Viscous Two-Dimensional Electron Liquid, Sci. Rep. 10, 7860 (2020).

[12] A. O. Govorov and J. J. Heremans, Hydrodynamic Effects in Interacting Fermi Electron Jets, Phys. Rev. Lett. 92, 026803 (2004). 
[13] A. I. Berdyugin, S. G. Xu, F. M. D. Pellegrino, R. K. Kumar, A. Principi, I. Torre, M. B. Shalom, T. Taniguchi, K. Watanabe, I. V. Grigorieva, M. Polini, A. K. Geim, and D. A. Bandurin, Measuring Hall Viscosity of Graphene's Electron Fluid, Science 364, 162 (2019).

[14] G. M. Gusev, A. D. Levin, E. V. Levinson, and A. K. Bakarov, Viscous Transport and Hall Viscosity in a TwoDimensional Electron System, Phys. Rev. B 98, 161303(R) (2018).

[15] M. J. M. de Jong and L. W. Molenkamp, Hydrodynamic Electron Flow in High-Mobility Wires, Phys. Rev. B 51, 13389 (1995).

[16] G. M. Gusev, A. D. Levin, E. V. Levinson, and A. K. Bakarov, Viscous Electron Flow in Mesoscopic TwoDimensional Electron Gas, AIP Adv. 8, 025318 (2018).

[17] E. I. Kiselev and J. Schmalian, Boundary Conditions of Viscous Electron Flow, Phys. Rev. B 99, 035430 (2019).

[18] We note that the terms "perfectly smooth," "perfect slip," and "no stress" conditions are equivalent and, hence, interchangeable.

[19] See Ref. [20] for similar whirlpool phenomena in graphene.

[20] F. M. D. Pellegrino, I. Torre, A. K. Geim, and M. Polini, Electron Hydrodynamics Dilemma: Whirlpools or No Whirlpools, Phys. Rev. B 94, 155414 (2016).

[21] C. Beenakker and H. van Houten, Quantum Transport in Semiconductor Nanostructures, Solid State Phys. 44, 1 (1991).

[22] Q. Shi, P. D. Martin, Q. A. Ebner, M. A. Zudov, L. N. Pfeiffer, and K. W. West, Colossal Negative Magnetoresistance in a Two-Dimensional Electron Gas, Phys. Rev. B 89, 201301(R) (2014).

[23] P. S. Alekseev and M. A. Semina, Ballistic Flow of TwoDimensional Interacting Electrons, Phys. Rev. B 98, 165412 (2018).

[24] F. M. D. Pellegrino, I. Torre, and M. Polini, Nonlocal Transport and the Hall Viscosity of Two-Dimensional Hydrodynamic Electron Liquids, Phys. Rev. B 96, 195401 (2017).

[25] L. Levitov and G. Falkovich, Electron Viscosity, Current Vortices and Negative Nonlocal Resistance in Graphene, Nat. Phys. 12, 672 (2016).

[26] I. Torre, A. Tomadin, A. K. Geim, and M. Polini, Nonlocal Transport and the Hydrodynamic Shear Viscosity in Graphene, Phys. Rev. B 92, 165433 (2015).

[27] A. Gupta, J. J. Heremans, G. Kataria, M. Chandra, S. Fallahi, G. C. Gardner, and M. J. Manfra, Hydrodynamic and Ballistic Transport over Large Length Scales in GaAs/AlGaAs, Phys. Rev. Lett. 126, 076803 (2021).

[28] H. Guo, E. Ilseven, G. Falkovich, and L. S. Levitov, Higherthan-Ballistic Conduction of Viscous Electron Flows, Proc. Natl. Acad. Sci. U.S.A. 114, 3068 (2017).
[29] G. F. Giuliani and J. J. Quinn, Lifetime of a Quasiparticle in a Two-Dimensional Electron Gas, Phys. Rev. B 26, 4421 (1982).

[30] P. S. Alekseev, Negative Magnetoresistance in Viscous Flow of Two-Dimensional Electrons, Phys. Rev. Lett. 117, 166601 (2016).

[31] T. Scaffidi, N. Nandi, B. Schmidt, A. P. Mackenzie, and J. E. Moore, Hydrodynamic Electron Flow and Hall Viscosity, Phys. Rev. Lett. 118, 226601 (2017).

[32] M.S. Steinberg, Viscosity of the Electron Gas in Metals, Phys. Rev. 109, 1486 (1958).

[33] R. Asgari, B. Davoudi, M. Polini, G. F. Giuliani, M. P. Tosi, and G. Vignale, Quasiparticle Self-Energy and Many-Body Effective Mass Enhancement in a TwoDimensional Electron Liquid, Phys. Rev. B 71, 045323 (2005).

[34] D. Q. Wang, J. C. H. Chen, O. Klochan, K. D. Gupta, D. Reuter, A. D. Wieck, D. A. Ritchie, and A. R. Hamilton, Influence of Surface States on Quantum and Transport Lifetimes in High-Quality Undoped Heterostructures, Phys. Rev. B 87, 195313 (2013).

[35] V. K. Arora and A. Naeem, Phonon-Scattering-Limited Mobility in a Quantum-Well Heterostructure, Phys. Rev. B 31, 3887 (1985).

[36] B. K. Ridley, The Electron-Phonon Interaction in QuasiTwo-Dimensional Semiconductor Quantum-Well Structures, J. Phys. C 15, 5899 (1982).

[37] T. Kawamura and S. D. Sarma, Temperature Dependence of the Low-Temperature Mobility in Ultrapure $\mathrm{Al}_{\mathrm{x}} \mathrm{Ga}_{1-\mathrm{x}} \mathrm{As} /$ GaAs Heterojunctions: Acoustic-Phonon Scattering, Phys. Rev. B 42, 3725 (1990).

[38] T. Kawamura and S. D. Sarma, Phonon-Scattering-Limited Electron Mobilities in $\mathrm{Al}_{x} \mathrm{Ga}_{1-x} \mathrm{As} / \mathrm{GaAs}$ Heterojunctions, Phys. Rev. B 45, 3612 (1992).

[39] L. Landau and E. Lifshitz, Fluid Mechanics (Elsevier, New York, 2013), Vol. 6.

[40] G. Mahan, Many-Particle Physics, Physics of Solids and Liquids (Springer, New York, 2000).

[41] D. Y. H. Ho, I. Yudhistira, N. Chakraborty, and S. Adam, Theoretical Determination of Hydrodynamic Window in Monolayer and Bilayer Graphene from Scattering Rates, Phys. Rev. B 97, 121404(R) (2018).

[42] H. H. Jensen, H. Smith, P. Wölfle, K. Nagai, and T. M. Bisgaard, Boundary Effects in Fluid Flow. Application to Quantum Liquids, J. Low Temp. Phys. 41, 473 (1980).

[43] A. Principi, G. Vignale, M. Carrega, and M. Polini, Bulk and Shear Viscosities of the Two-Dimensional Electron Liquid in a Doped Graphene Sheet, Phys. Rev. B 93, 125410 (2016).

[44] D. Tong, Lecture Notes on Kinetic Theory, https://www .damtp.cam.ac.uk/user/tong/kinetic.html. 